$\mathrm{DOE} / \mathrm{NT} / 41970$

\title{
MEMS CHIP CO2 SENSOR FOR BUILDING SYSTEMS INTEGRATION
}

Final Technical Report

Contract number DE-FC26-04NT41970

Issued on:

September 14, 2005

Issued by:

Anton Carl Greenwald

Ion Optics, Inc.

411 Waverley Oaks Road, Suite 144,

Waltham, MA 02452-8484

This report was prepared as an account of work sponsored by an agency of the United States Government. Neither the United States Government nor any agency thereof, nor any of their employees, nor any of their contractors, subcontractors or their employees, makes any warranty, express or implied, or assumes any legal liability or responsibility for the accuracy, completeness, or any third party's use or the results of such use of any information, apparatus, product, or process disclosed, or represents that its use would not infringe privately owned rights. Reference herein to any specific commercial product, process, or service by trade name, trademark, manufacturer, or otherwise, does not necessarily constitute or imply its endorsement, recommendation, or favoring by the United States Government or any agency thereof or its contractors or subcontractors. The views and opinions of authors expressed herein do not necessarily state or reflect those of the United States Government or any agency thereof.

subject categories 54, Environmental Science, 47 Other instrumentation (non-nuclear) 


\section{ABSTRACT}

The objective of this research was to develop an affordable, reliable sensor to enable demand controlled ventilation (DCV). A significant portion of total energy consumption in the United States is used for heating or air conditioning (HVAC) buildings. To assure occupant safety and fresh air levels in large buildings, and especially those with sealed windows, HVAC systems are frequently run in excess of true requirements as automated systems cannot now tell the occupancy level of interior spaces. If such a sensor (e.g. thermostat sized device) were available, it would reduce energy use between 10 and $20 \%$ in such buildings.

A quantitative measure of "fresh air" is the concentration of carbon dioxide $\left(\mathrm{CO}_{2}\right)$ present. An inert gas, $\mathrm{CO}_{2}$ is not easily detected by chemical sensors and is usually measured by infrared spectroscopy. Ion Optics research developed a complete infrared sensor package on a single MEMS chip. It contains the infrared (IR) source, IR detector and IR filter. The device resulting from this DOE sponsored research has sufficient sensitivity, lifetime, and drift rate to meet the specifications of commercial instrument manufacturers who are now testing the device for use in their building systems. 


\section{TABLE OF CONTENTS}

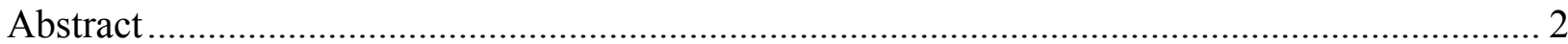

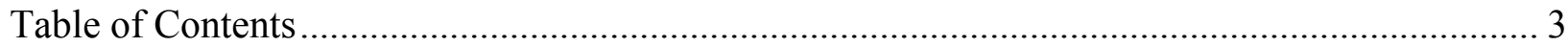

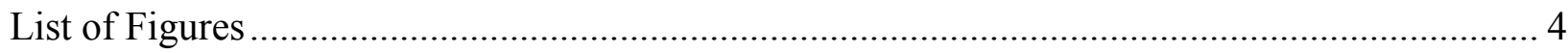

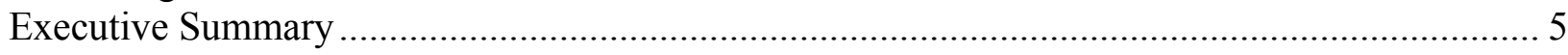

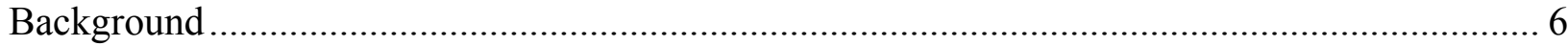

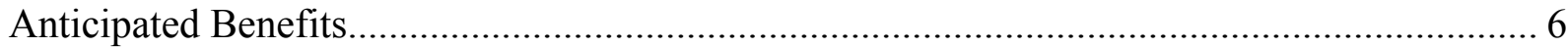

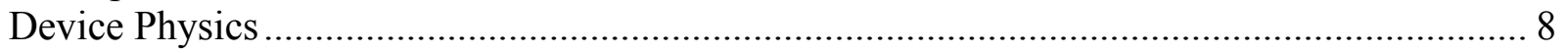

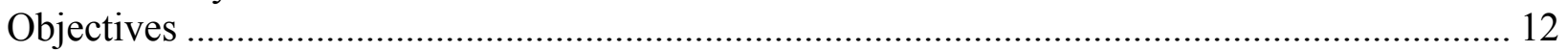

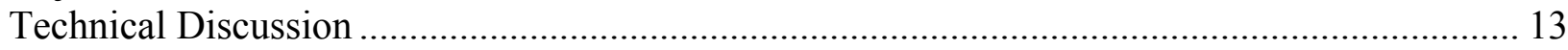

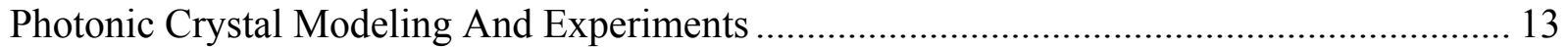

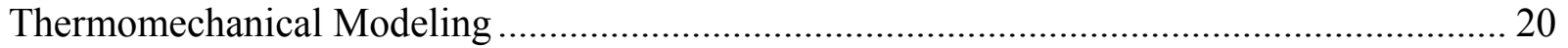

Optimization Of Diffusion Barrier........................................................................... 23

Optimization Of Metallization ...................................................................................... 23

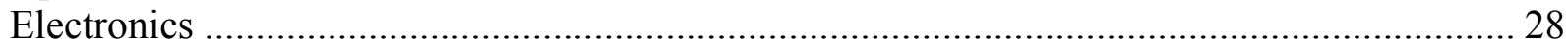

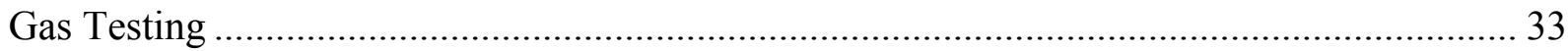

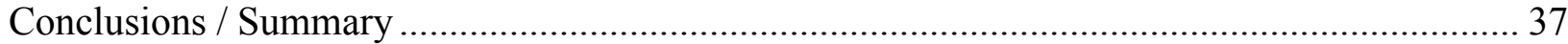

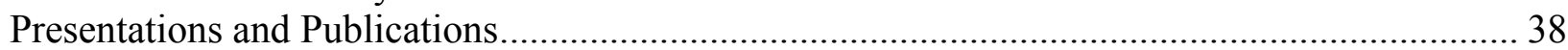

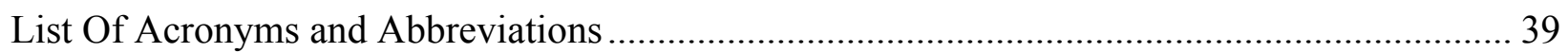

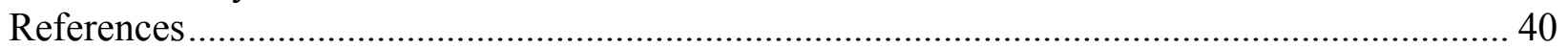




\section{LIST OF FIGURES}

Figure 1 Principal of operation of the MEMS carbon dioxide chip. 9

Figure 2 Interaction of Photonic Crystal with light. 9

Figure 3 Measured emission from photonic crystal as a function of etch depth in silicon. 10

$\begin{array}{lll}\text { Figure } 4 \text { Diagram of prototype CO2 sensor chip. } & 11\end{array}$

$\begin{array}{lll}\text { Figure } 5 \text { Prototype CO2 sensor in leadless chip carrier package. } & 11\end{array}$

$\begin{array}{lll}\text { Figure } 6 & \text { Emission peak from thermally excited 2-D photonic crystal. } & 13\end{array}$

$\begin{array}{lll}\text { Figure } 7 & \text { Reflection data comparing measured results to model calculations. } & 14\end{array}$

$\begin{array}{lll}\text { Figure } 8 \text { Cross-section of 2-D photonic crystal and SEM microphotograph of surface. } & 14\end{array}$

$\begin{array}{lll}\text { Figure } 9 \text { Geometrical description of problem. } & 14\end{array}$

Figure 10 Model calculation of reflectance as a function of wavelength from photonic $\begin{array}{ll}\text { crystal with varying silicon resistance. } & 15\end{array}$

Figure 11 Measured reflection from 2-D photonic crystal used for $\mathrm{CO} 2$ detector. 16

Figure 12 Measured reflection from 2-D photonic crystal used for H2O detector. 17

Figure 13 Experimental measurements showing effect of etch depth in silicon on reflection from photonic crystal structure.

$\begin{array}{ll}\text { Figure } 14 \text { Reflection as a function of wavelength (s-polarization). } & 18\end{array}$

$\begin{array}{ll}\text { Figure } 15 \text { Reflection as a function of wavelength (p-polarization). } & 18\end{array}$

$\begin{array}{ll}\text { Figure } 16 \text { SEM photograph of cleaved test sample fabricated for CO2 detection. } & 19\end{array}$

Figure 17 Comparison of theory to experiment 6 micron etch. 19

Figure 18 Comparison of theory to experiment 500nm etch (P polarization). 20

Figure 19 Comparison of theory to experiment 500nm etch (S polarization). 20

Figure 20 Mechanical distortion of heated MEMS device. 22

Figure 21 Thermal profile of MEMS chip at a pressure of 100mtorr. 22

Figure 22 Thermal profile of MEMS chip at a pressure of 10mtorr. 22

Figure 23 Emission from photonic crystals with varying thickness of Platinum. 24

Figure 24 Stability of high temperature annealed TaPt and TiPt. 25

Figure 25 Drift per day for Ti-Pt and Ta-Pt metal layers. 25

Figure 26 Micrograph before and after annealing at $800^{\circ} \mathrm{C}$. 26

Figure 27 View of the annealed Ti-Pt film. 26

Figure 28 Cross sectional TEM micrograph of an annealed Ta-Pt metallization. 26

Figure 29 Scanning electron microscope image of bus-bar on support arm. 27

$\begin{array}{ll}\text { Figure } 30 \text { Wheatstone bridge circuit for drive and readout of gas sensor. } & 29\end{array}$

Figure 31 Prototype $\mathrm{CO}_{2}$ sensor, packaged in LCC. 29

Figure 32 Schematic diagram of optimal control scheme using 12 IC's. 31

Figure 33 Drift of baseline at start of this project and at end of program. 32

Figure 34 Drift of signal with time. 32

Figure 35 Ion Optics SensorChip ${ }^{\text {TM }}$ precision for CO2. 34

Figure 36 The effect of water vapor on $\mathrm{CO} 2$ measurements with breadboard sensor. 35

Figure 37 Signal from $\mathrm{CO}_{2}$ sensor for potential interferent hydrocarbons. 36

Figure 38 Signal from alcohol as a potential interferent. 36

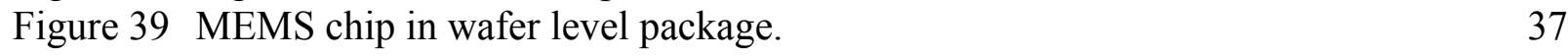




\section{EXECUTIVE SUMMARY}

At the conclusion of work on this project, Ion Optics fabricated and delivered to prospective OEM customers prototype quantities of a MEMS (micro-electronic mechanical system) carbon dioxide sensor for indoor air quality measurement. This device is significantly smaller and consumes less power than available technology. At lower cost and enabling wireless installation, it is expected to be adopted for automated HVAC controls in commercial buildings. Potential energy savings result from adjusting ventilation flow and mixing with outdoor air according to occupation demand, instead of arbitrary timed flow to assure meeting minimum air quality standards at maximum occupancy.

At the start of work on this project Ion Optics had demonstrated the feasibility of combining an infrared source, filter and detector on one MEMS device that could detect carbon dioxide. Potential customers for this application were interested, but noted that the device did not meet necessary specifications. With the financial assistance award from DOE:

- Detailed surface structure was improved, increasing signal to noise ratio and yield

- Materials choices were optimized improving long term drift

- Electronics were improved zeroing thermal drift

- Revised devices now meet all required specifications

At no cost to DOE, Ion Optics is working to improve the packaging of this MEMS device and current cost estimates are within customers' desired range. The estimated delay time between customers' evaluation and production is expected to be about 18 months.

Additional applications of the device were discovered during this research. The surface structure (2-D photonic crystal) of the MEMS chip is a very efficient infrared emitter. Potential applications will require a revised chip design, but as the final package and electronics are much simpler than that required for the gas sensor, time to market is short and significant revenue is anticipated in 2005 .

Other benefits of this work include contributions to the theory of photonic crystals and especially devices utilizing surface plasmon oscillations. 


\section{BACKGROUND}

\section{Anticipated Benefits}

Ion Optics expects to market infrared carbon dioxide $\left(\mathrm{CO}_{2}\right)$ sensors to manufacturers of indoor ventilation control systems. In this section we will discuss the advantages of such sensors for controlling building ventilation, and advantages to the nation with widespread use of such technology. $1,2,3$

\section{Energy Savings with Sensor Based Demand Controlled Ventilation}

Building ventilation technologies and sensors are an important component in national energy use. Existing HVAC in large buildings are adjusted to maintain constant temperature with minimum required ventilation. Ventilation rates are being increased to mitigate indoor air quality (IAQ) concerns. Prior code requirements such as ASHRAE standard 62-1989 listed minimum ventilation rate per occupant, and these numbers were derived from assumptions needed to maintain $\mathrm{CO}_{2}$ concentrations below $1,000 \mathrm{ppm}$. Variable pollutant sources not related to human occupancy may alter these codes.

Energy savings from $\mathrm{CO}_{2}$ based demand controlled ventilation result from employing ASHRAE target ventilation rates based on actual building occupancy rather than assumed maximum occupancy. A typical timer control strategy for an office building activates full ventilation based on design occupancy at the beginning of an occupancy period and turns it off at the end of the day. While using the right $\mathrm{CO}_{2}$ demand controlled ventilation (DCV) strategy, it is possible to ensure that $\mathrm{cfm} /$ person ventilation is maintained based on actual occupancy. Consider the case for an auditorium or movie theater. Ventilation strategy would run air flow during expected occupation times for about $60 \%$ seating capacity. This would be either more or less than desired values and only a sensor-based approach could minimize energy usage while maintaining adequate comfort levels. Potential energy savings of the DCV approach have been estimated to be as high as $25 \%$ in office buildings. Potential customers for Ion Optics' sensors expect energy savings between 10 and $20 \%$.

Energy savings vary compared to type of technology being replaced - simple timers that do not recognize holidays perform poorly when compared to computerized timers. Energy savings are even greater if the outside ventilation rates are higher than design ventilation rates based on maximum occupancy. This can often occur when building operators set minimum air intakes to an "eye-ball" setting of $20 \%$ rather than measuring airflow and not understanding that damper position is not indicative of actual air flow. It is important to note that in these situations $\mathrm{CO}_{2}$ levels will automatically determine the appropriate airflow for the actual occupancy of the space or building.

Why is measuring $\mathrm{CO}_{2}$ particularly important? There are many sources of pollution in a building and most, but not all, can be related to human occupancy. Studies have shown a fair correlation of $\mathrm{CO}_{2}$ levels to the number of people in a building, and to the amount of many other pollutants. While infrared heat sensors can measure occupancy directly, and have been used in tests for DCV, a smaller number of gas sensors for $\mathrm{CO}_{2}$ (in exhaust flows) can measure the occupancy rate for a large building zone with many rooms. Other energy benefits from a $\mathrm{CO}_{2} \mathrm{DCV}$ strategy include: 
- A $\mathrm{CO}_{2}$ control strategy will include air from infiltration, open windows or other sources of outside air as contributing to the overall ventilation requirements, eliminating any redundant ventilation that might otherwise be conditioned and delivered by the mechanical system.

- Used in conjunction with variable air volume systems (VAV) and other multiple zoned systems, ventilated air can be controlled not only at the air intake but also in individual zones. This approach optimizes ventilation efficiency in particular zones based on actual occupancy and may also allow the use of air from other lower occupancy zones to provide fresh air to zones where a ventilation demand exists.

Assuming that a $25 \%$ energy use reduction is a conservative number and typical of the average national energy reduction possible, energy savings are detailed below. It is important to note that current market penetration of DCV as a ventilation control strategy is less than $0.03 \%$ of the total potential market. Virtually all commercial buildings are candidates for this approach particularly if the same device can also provide humidity control.

\% Energy Savings X National Energy X Maximum Possible = National Total Consumption for End Market Penetration Energy Savings

For ventilation: $25 \%$ X 0.54 Quads X 100\% $=0.135$ Quads

National total carbon savings are calculated as follows:

National Total X Energy To Carbon = National Total

Energy Savings Factor Carbon Savings

1.35 Quads X 15.19 Kg/MMbtu's $=2.05 \times 10^{6}$ metric tons of carbon

Environmental benefits of $\mathrm{CO}_{2}$ based demand controlled ventilation include:

- Unnecessary over ventilation of buildings is reduced thereby reducing operational energy requirements.

- While ventilation levels are reduced with demand controlled ventilation, target per-person ventilation rates recommended by ASHRAE can be maintained at all times ensuring acceptable air quality.

- $\mathrm{CO}_{2}$ demand controlled ventilation can be used in humid climates to limit the introduction of high humidity outdoor air to that which is only required for acceptable air quality. The potential of bringing in excessive humid air that can adversely impact comfort and air quality (through the growth of mold) is minimized.

- The amount of ventilation delivered to a particular zone in a multi-zone building has generally been empirically determined because the only place that the volume of outside air could be measured is in the outdoor intake. $\mathrm{CO}_{2}$ can be used as a measure of the actual efficiency of fresh air distribution by zone. This allows for more effective control of ventilation to ensure acceptable air quality throughout a building.

- Elevated outside $\mathrm{CO}_{2}$ levels can be indicative of the presence of combustion fumes. $\mathrm{CO}_{2}$ sensors can also be used to periodically close outdoor air intakes for acceptable indoor air conditions.

Important application examples include movie theaters and schools. During occupied hours, populations are dense and ASHRAE 62-1989 specifies $15 \mathrm{cfm}$ per person outside air for acceptable levels of indoor air quality. A properly functioning $\mathrm{CO}_{2}$ and humidity sensor would 
provide a means of HVAC control that would achieve both acceptable IAQ and proper levels of indoor air conditioning to maintain an acceptable comfort zone for the occupants. This is especially import for movie theaters where populations can vary from less than $10 \%$ to $100 \%$ occupancy in one business day. From a first cost and energy consumption standpoint, it is not practical to design a theater using the assumption of $100 \%$ occupancy at all times. Many times, the designers will assume an average occupancy of $60 \%$ during business hours. When movie theater populations rise to $100 \%$, unacceptable levels of $\mathrm{CO} 2$ result, in some instances, as high as $2000 \mathrm{ppm}$. A $\mathrm{CO}_{2}$ sensor would maintain the indoor air quality at acceptable levels of $\mathrm{CO}_{2}$ while cycling the HVAC system for lower than $60 \%$ occupancy times to maintain indoor space comfort. Similarly, for schools the HVAC system and its bypasses would be controlled to provide for the acceptable IAQ of the students and economize the vapor-compression system use to provide space comfort at minimal energy expenditure.

\section{Device Physics}

A summary of device operation is described in Figure 1 below. A heated filament is suspended by thin, thermally isolating arms from a silicon frame providing electrical connections to external circuits. The surface of this silicon filament is covered with a very thin layer of patterned metal. The surface structure acts as a 2-D photonic crystal with properties significantly different from those of a black body. When heated, most emitted energy is confined to a narrow wavelength band centered on the absorption line of $\mathrm{CO}_{2}$. This radiant energy is also highly directional, normal to the surface, and passes through the IR transparent package. The light traverses a "gas cell" and is reflected and focused back onto the filament by a spherical mirror. The photonic crystal preferentially absorbs light in the same wavelength band it emits. If a vapor species in the gas cell absorbs some of the radiation, the temperature of the filament will be altered. Because the resistance of the filament changes with temperature, the signal of gas absorption is detected as a current change when heating at constant voltage.

Two innovative aspects of this design were patented by Ion Optics prior to the start of this DOE program. One patent was issued on the design of a 2-D surface pattern to control the emitted wavelength. ${ }^{4}$ The second issued patent is on the use of a thin filament as a combined source and detector, a hot bolometer. ${ }^{5}$

Detailed theory of operation of the photonic crystal (Figure 2) to operate as a selective emitter was published separately. ${ }^{6}$ Without going through the detailed equations, the energy in the heated silicon filament couples to plasmon modes at the metal-silicon interface. Only certain frequencies can couple and this coupling is possible only because of the patterned structure. The plasmons modes on one side of the thin metal film couple to plasmon modes on the other side (metal-air interface) and the energy can be coupled to photons emitted from the surface, also enabled by the surface pattern. While other published papers have suggested that a very thin patterned metal layer, by itself, can function as an optical filter, that is not what happens in this case. $^{7}$ If the underlying silicon is not patterned or too thin, the characteristic peaked emission is destroyed (Figure 3). 


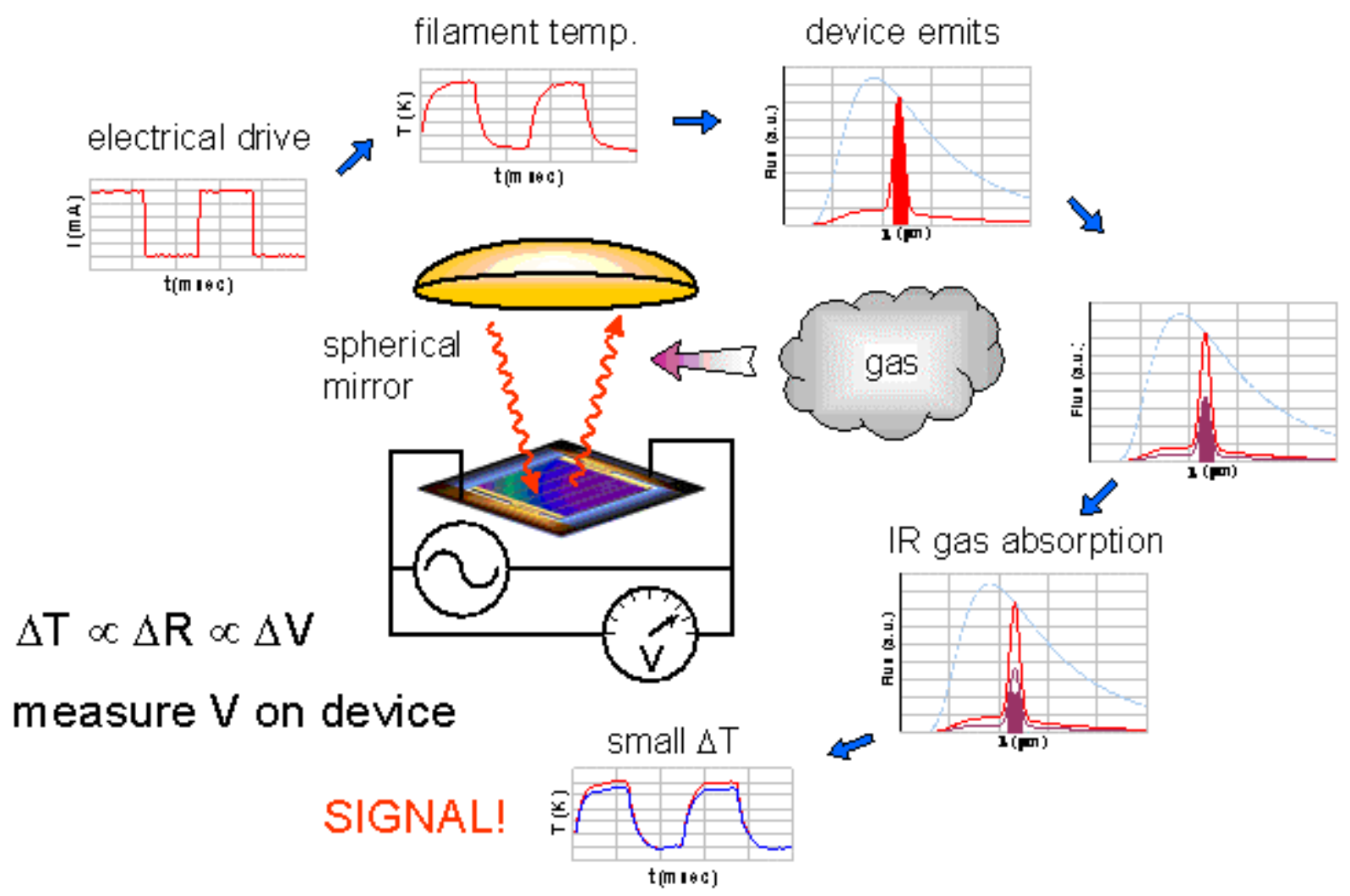

Figure 1 Principal of operation of the MEMS carbon dioxide chip. An unsupported MEMS filament is electrically heated to $325^{\circ} \mathrm{C}$ by a pulsed electrical signal. Surface structure (photonic crystal) limits emitted radiation to a narrow peak with low level broad background. Light passes through the gas sample to be measured and is reflected back to the chip. Because the filament is thin, the change in reflected light due to absorption in the gas causes a change in temperature that can be measured. Specificity is attained through a narrow peak at designed wavelength.

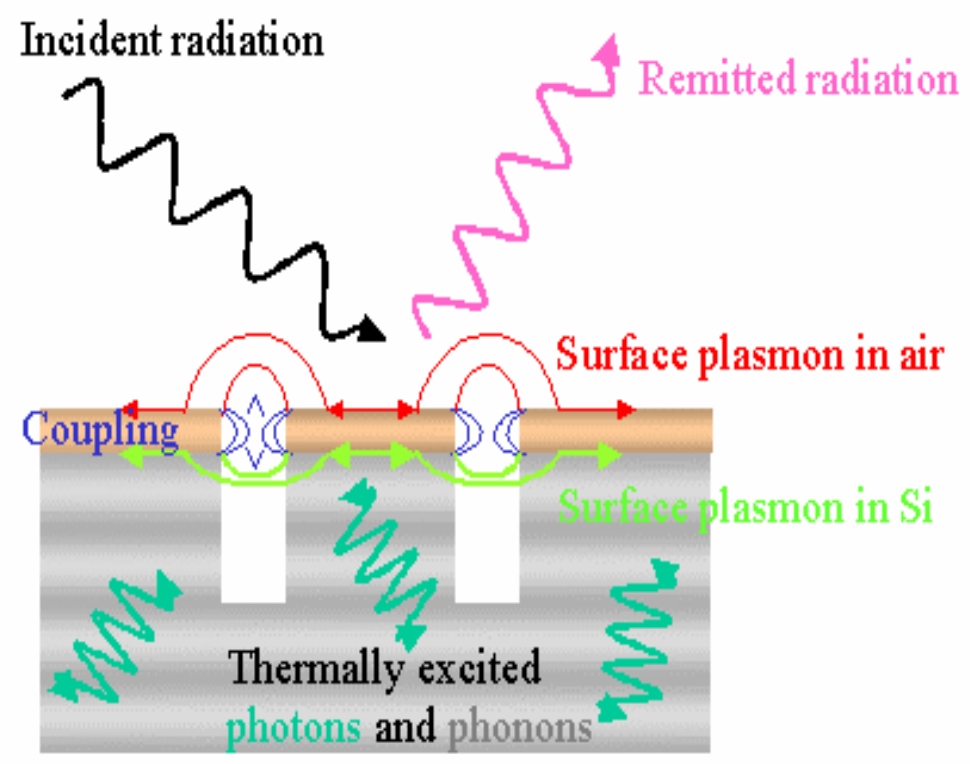

Figure 2 Interaction of Photonic Crystal with light. Bottom support is silicon and top layer is metal. There is a thin diffusion barrier (not shown) between the metal and silicon. 


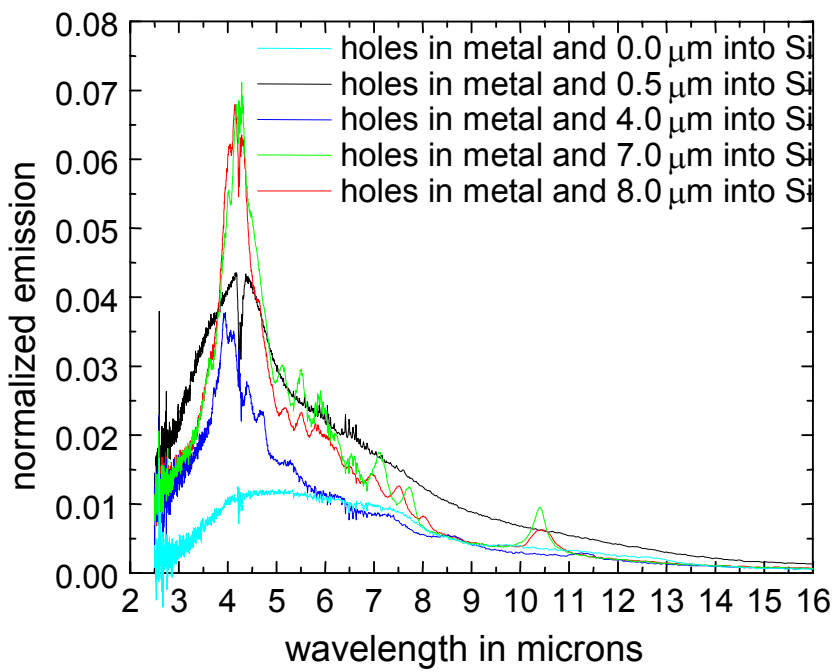

Figure 3 Measured emission from photonic crystal versus absorbed wavelength for various etch depths in silicon. If silicon not etched (0 microns) emission is that of a black body with low emissivity. Emission peak is low for thin silicon etch of 0.5 to 4.0 microns. Emission was maximized for an etch depth of about 8 microns. ${ }^{8}$

The supporting silicon membrane is at least ten microns thick with the photonic crystal pattern (holes) etched about 8 microns deep (Figure 3). Data in Figure 3 was discovered by Ion Optics in the 18 months between the time the proposal was written and the start of work on this project. It forced changes to the original planned statement of work and drives many other design choices. Original plans were to use polycrystalline silicon for the membrane material. However, poly-silicon films cannot be deposited to this thickness with low stress and smooth surface structure, so that the MEMS device is formed from bonded silicon-on-insulator (SOI) material. As the filament is now single crystal silicon material, it is mechanically stronger than poly, but it does not have a monotonically increasing resistance as a function of temperature. In the temperature range of interest, single crystal silicon has a resistance maximum and then drops, due to free carrier generation, as the temperature is further increased. Prior experiments with the MEMS sensor tested use of the silicon support as heater and/or sensing element. ${ }^{9}$ Stability was improved and electronic noise was reduced by heating the device with the metal film as well as using that metal film as the sensing resistor.

The area of the heated filament is larger than that used in other types of MEMS gas sensors. ${ }^{10}$ This is required by optical and thermo-mechanical design. A small filament would require great care in alignment of the MEMS component and the mirror and the cost of that change exceeds that cost of using a larger die. Because the thickness of the filament (10 microns) is much greater than the thickness of other sensors ( 0.1 micron), the supporting arms must be thicker for mechanical stability. This increases the parasitic thermal conduction losses down the supports. To maintain signal to noise ratio sufficient for required precision, the area of the heated filament is larger increasing the ratio (change of heat flux due to gas absorption) to (parasitic heat flux losses).

The MEMS sensor has two filaments of equal size (Figure 4). The original design called for one filament to be used as a reference signal at 3.9 micron wavelength for the same reason most NDIR gas sensors use a reference signal to compensate for variations in source output, obscuration, temperature and pressure differences, etc. When this proposal was written, it was believed that this second filament could be used as a water vapor sensor. Practical optical design prevents either use. To focus the light from one filament back onto the same filament for correct operation prevents use of a spherical mirror. The optical efficiency of alternate designs, micro- 
retro-reflectors, was only $10 \%$ compared to the $30 \%$ now achieved. Optical efficiency loss would reduce precision. The second filament is blanked off and used for thermal compensation.

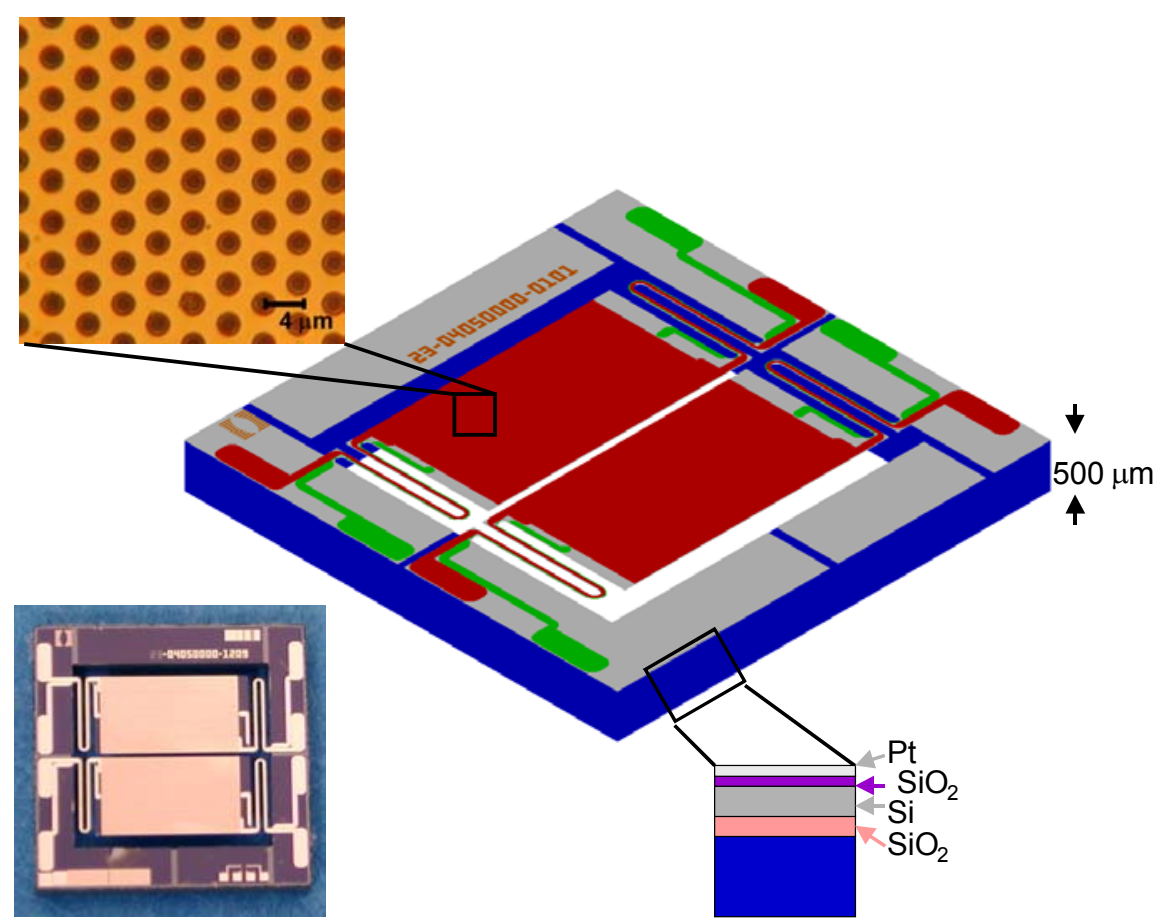

Figure 4 Prototype $\mathrm{CO}_{2}$ sensor chip is $5 \mathrm{~mm}$ square. The silicon base is a standard wafer $0.5 \mathrm{~mm}$ thick. A bonded SOI layer typically over 10 microns thick is added. Two filament heaters are shown in red and high current bus-bar is in red. Base silicon and oxide are removed from underneath the filaments so these structures are unsupported, except for " $U$ " shaped leg, and are thermally isolated. A second wire on either side of filament (green) is used to measure potential across sensor. Picture of actual prototype (lower left) shows additional test patterns on supporting frame.

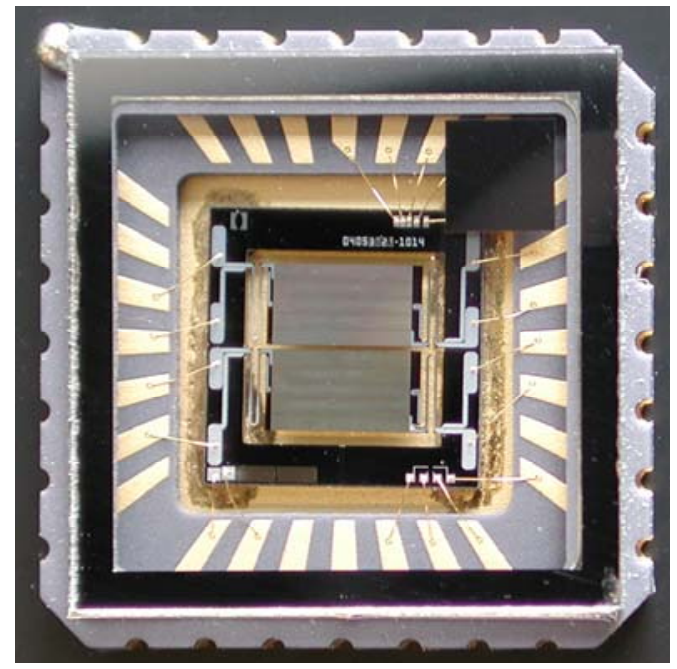

Figure 5 Prototype $\mathrm{CO}_{2}$ sensor (5mm square) in leadless chip carrier package (15mm square) configured for optical testing. Internal getter material is visible upper right hand corner. Two filaments can be seen in center; normally one is covered for gas sensor operation ). 


\section{OBJECTIVES}

The overall objective of this project was to fabricate a prototype indoor air sensor that can be delivered to a large manufacturer of building controls for their internal testing. This objective was met. Preferred specifications are volume under $100 \mathrm{cc}$, average power draw less than $1 \mathrm{~mW}$, detection of carbon dioxide to an accuracy of 50ppm, and cost less than $\$ 15$ per unit. To achieve this objective, Ion Optics designed, fabricated and tested modifications to its proprietary microopto-electronic system (MEMS) sensor chip and electronics.

Ion Optics achieved the following improvements in sensor performance during the course of this project comparing the original laboratory demonstration experiment with the final prototype suitable for distribution.

- $\quad$ The minimum sensitivity and precision of the sensor was improved from $\pm 1000 \mathrm{ppm}$ or $\pm 0.1 \%$ to $\pm 50 \mathrm{ppm}$ volume concentration of $\mathrm{CO}_{2}$ in air.

- $\quad$ The temperature drift of the sensor was reduced from over $100 \mathrm{ppm} \mathrm{CO}_{2}$ concentration per degree Celsius change in ambient temperature to zero within limits of measurement instruments.

- $\quad$ The drift with time of the sensor was reduced from over $1 \% \mathrm{CO}_{2}$ concentration per day to less than 50ppm per day.

- $\quad$ MEMS power draw during operation was reduced to $60 \mathrm{~mW}$ (less than $1 \mathrm{~mW}$ average with expected low duty cycle operation).

- $\quad$ The time required to take a reading from turn-on was reduced to less than two seconds.

Technical objectives were to:

- Create a computer model that could match experimental results

- Use this model to ascertain desired material properties

- Fabricate devices with silicon of different properties (e.g. resistivity) to verify model and improve device performance

- Determine optimum insulator thickness

- Determine optimum metal composition and thickness

- Improve drive electronics - reduce drift and increase signal-to-noise ratio

Although some technical parameters were far different in the final prototype than anticipated in the original proposal, our overall goals were successfully achieved. The total volume per unit including electronics is under 100cc and expected cost per unit should be less than the $\$ 15$ goal with improved packaging (see Conclusions/Summary). For comparison, existing technology is larger, requires $1 \mathrm{~W}$ when operating or $150 \mathrm{~mW}$ when idle, and costs over $\$ 100$, which explains why is has not been widely adopted for the proposed application. 


\section{TECHNICAL DISCUSSION}

\section{Photonic Crystal Modeling and Experiments}

The measured optical emission from the photonic crystal structure designed for detection of $\mathrm{CO}_{2}$ is shown in Figure 6. This is a vacuum measurement without window - the sapphire window in delivered prototype units cuts off all light with a wavelength greater than 6 microns. Even with this cut-off, the existing emission overlaps absorption peaks of water vapor and hydrocarbons. If the peak width can be reduced, it would reduce interference and significantly improve precision. For comparison, very narrow line lasers have detection limits with similar other instrumentation as low as $0.1 \mathrm{ppm}$. Is this possible for photonic crystal based detectors? The expanded scale shows that the main peak is comprised of several sub-peaks that have been related to different modes of the photonic crystal structure. If the photonic crystal surface structure can be designed to suppress alternate modes, the emission peak can be made very narrow.
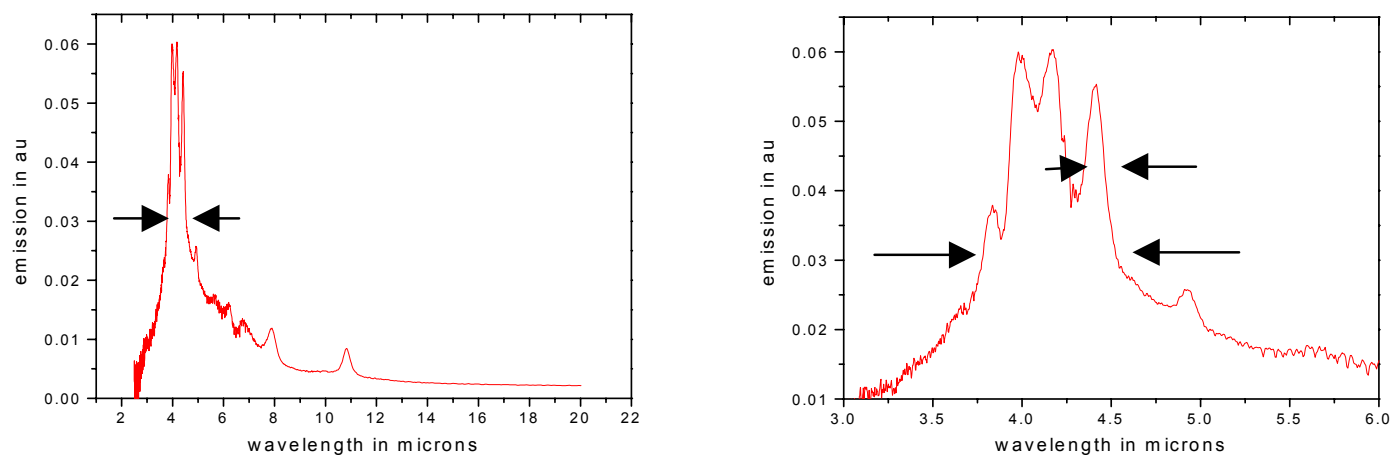

Figure 6 Emission peak from thermally excited 2-D photonic crystal shown on an expanded scale (right) illustrating sub-peaks with very narrow FWHM. ${ }^{8}$

A computer simulation was developed to predict photonic crystal behavior by Dr. William Schaich of the University of Indiana at Bloomington. It uses a highly magnified detail of one cell of the surface structure, assuming infinite symmetry, to predict photonic crystal behavior. The calculation was performed on a "super-computer" (e.g. very high speed multi-processor system). Dr. Schaich had been working with Ion Optics prior to the start of this contract as our problem presented an exciting new theoretical problem. For this work, he was given a subcontract to model the change in resistance of the silicon substrate, oxide, metal and silicon thickness effects. After the start of this contract Ion Optics received another grant from NSF to study theoretically and experimentally emission from 2-D photonic crystals so as to enable coherent emission from same. ${ }^{11}$ NSF funded studies of the detailed structure of the boundaries between the different materials, the shape of etched pattern or air hole (Figure 2) and geometrical arrangement of these patterns. The statement of work of this NSF program is very different than that of the DOE grant, but results from this second program may improve future generations of the gas sensor.

The first part of the modeling task was to show how the model can predict actual experimental results. This data is shown in Figure 7. The model gives reflection data so this figure plots reflection from the same type sample as used for emission in Figure 6. Agreement on major features is good. See Figure 17 for discussion on differences of fine structure. 


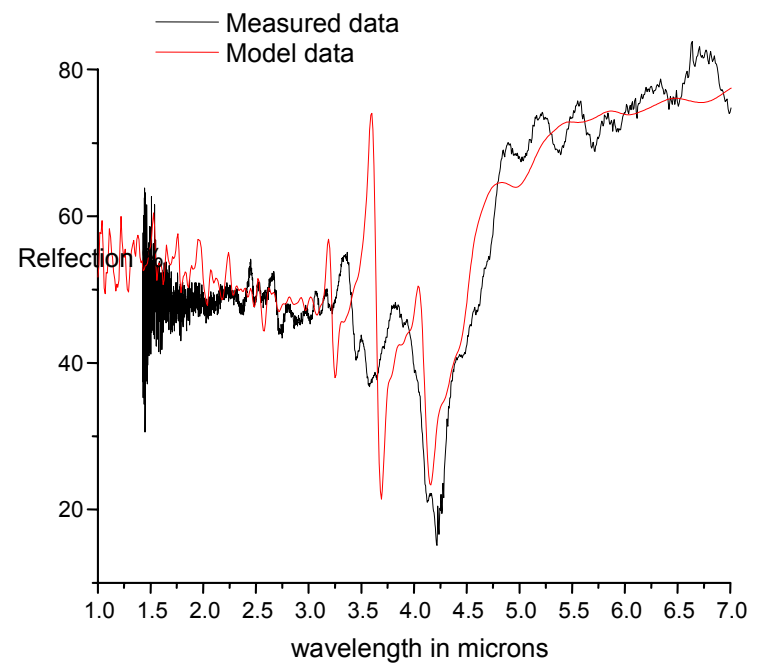

Figure $7 \quad$ Reflection data for sample similar to that of Figure 6 comparing measured results (black line) to model calculations (red line). Angle of incidence was $10^{\circ}$. Alignment to surface geometry at $\varphi=0$ (Figure 9), s-polarization.

The model geometry (Figure 8) for this data was a circular hole 6 microns deep and 2.1 microns diameter. The boundary conditions assume that there is hexagonal symmetry with center to center spacing of the holes at 4.2 microns. Silicon resistance was high $(3-5 \mathrm{ohm}-\mathrm{cm})$. The top layer for the model was aluminum 0.1 micron thick. Actual experiment uses platinum but sufficient material data was not available for this model at the time of this work. A thin diffusion barrier between the metal layer and silicon was included in this calculation though not shown in Figure 8. Electromagnetic fields were calculated one frequency (wavelength) at a time for many intervals over the range 2 to 10 microns. The input radiation was assumed to incident at an angle of $10^{\circ}$ from the normal, s polarized (electric field in plane containing incident and reflected light ray) and aligned to a main axis of the geometrical hole pattern (Figure 9). The experimental data covers a small range of angles.
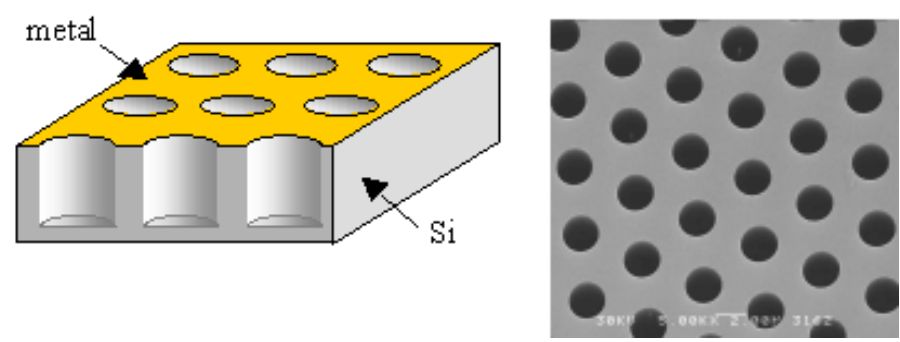

Figure 8 Cross-section diagram of existing structure for 2-D photonic crystal and SEM microphotograph of surface.

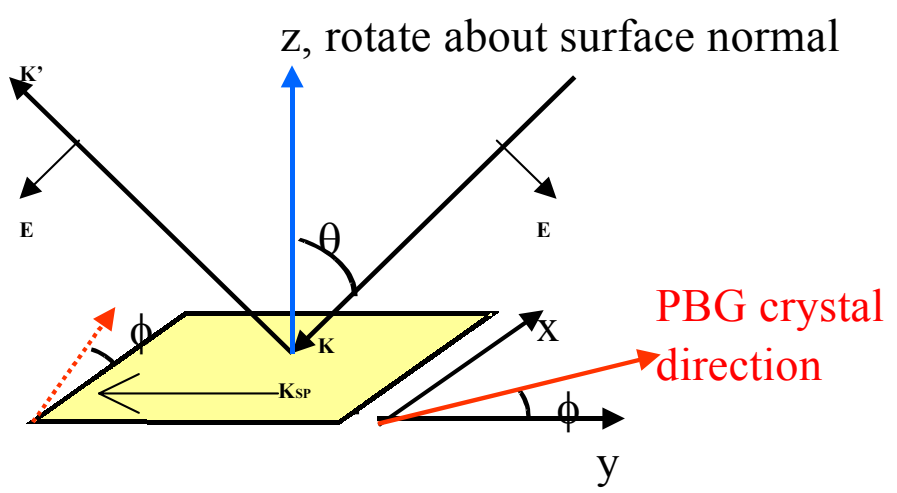

Figure 9 Geometrical description of problem. Angle of incidence is theta and phi is angle relative to surface geometry. For s-polarization the electric field is in the plane containing the incident and reflected ray. For p-polarized light the electric field is perpendicular to this plane. 
After verifying the model, calculations were made to compare the effects of silicon resistance and depth of the etched pattern (holes). Calculations were matched to experimental data. Key conclusions were that deep holes were required (typically $\geq 8$ microns) and low resistance silicon smoothed out fine structure at both long and shorter wavelengths without reduction of the major emission peak. [Modeling calculations are being continued in the NSF program ${ }^{11}$ that is examining changes in the geometry, now just an array of round holes. We have not yet found a means to separate the sub-peaks of the main emission line.]

Data in Figure 10 show results for the geometry of Figure 8 as designed to detect $\mathrm{CO}_{2}$. The model included a diffusion barrier between the top metal layer and underlying silicon, thickness of all films corresponding to experimental values. The vertical scale is reflection as a fraction from 0.0 to 1.0 and the horizontal scale is wavelength in microns. Suppression of secondary oscillation modes is key to narrowing the emission band and increasing sensitivity.

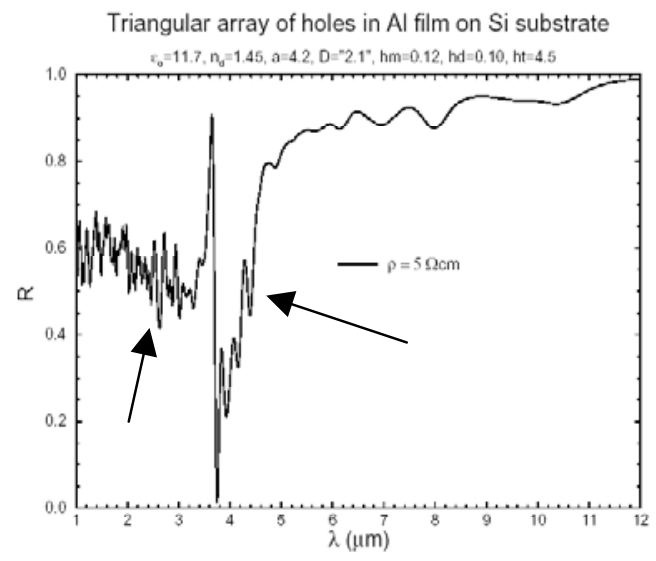

Triangular array of holes in Al film on Si substrate

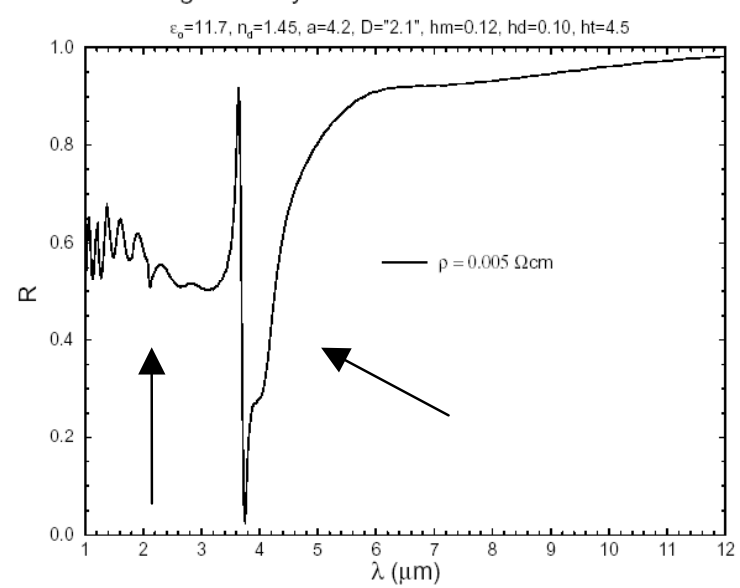

Triangular array of holes in Al film on Si substrate

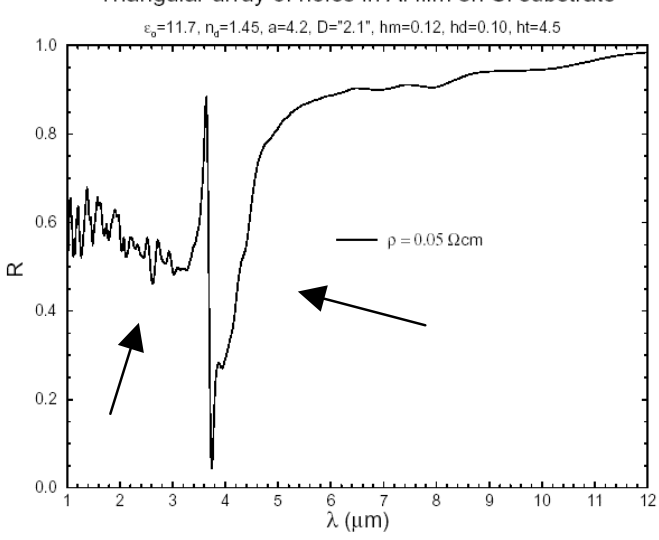

Figure 10 Model calculation of reflectance as a function of wavelength from photonic crystal with varying silicon resistance. Top left

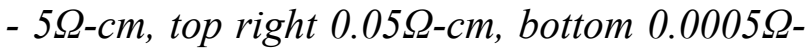
cm. Higher conductivity smoothes curves reducing or eliminating secondary peaks.

These predictions were tested by experiment. The photonic crystal structure was reproduced on a solid silicon wafer $0.5 \mathrm{~mm}$ thick. Prior experiments showed that there is no discernable difference between the reflection-absorption-emission data for this structure compared to an identical structure on a 10 micron thick silicon membrane. Data presented in Figure 11 are for a $\mathrm{CO}_{2}$ detector with designed hexagonal periodicity of 4.2 microns and hole diameter of 2.1 microns. The silicon substrate is doped N-type (phosphorus) and is of $\langle 100\rangle$ orientation. The three samples shown were:

$0.001-0.005 \mathrm{ohm}-\mathrm{cm}$ fabricated at Cornell $0.005-0.02 \mathrm{ohm}-\mathrm{cm}$ fabricated at Cornell 3-5 ohm-cm fabricated at JPL 


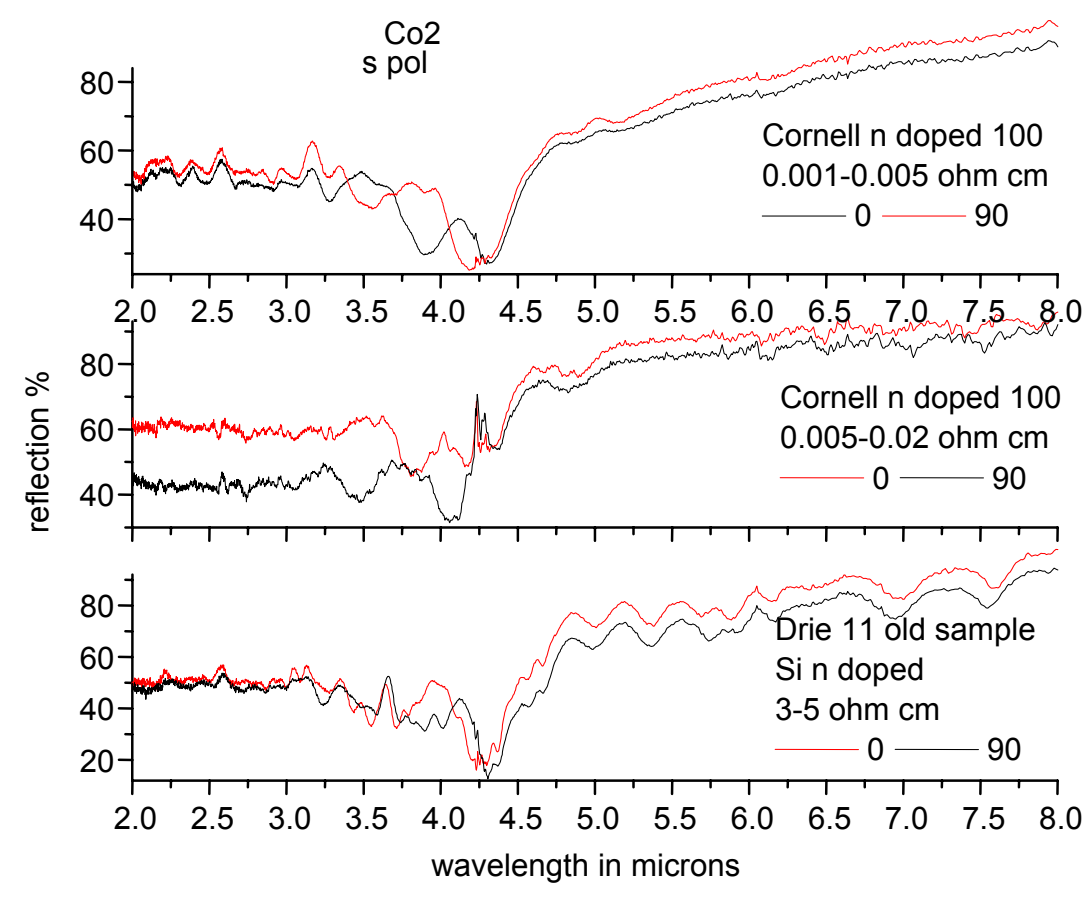

Figure 11 Measured reflection from 2-D photonic crystal used for $\mathrm{CO}_{2}$ detector as a function of the resistance of supporting silicon. Data was taken at two angles $\left(0^{\circ}\right.$ and $\left.90^{\circ}\right)$ relative to surface pattern at $10^{\circ}$ angle of incidence. Low resistance silicon suppresses secondary resonances as predicted by model.

One could see the gradual appearance of secondary resonances at short and longer wavelength (compare to the resonance wavelength), that gradually increase in frequency and amplitude as predicted by theory. Data in Figure 12 are identical except that the surface structure is designed to detect water vapor at 6.1 micron absorption line. Resonance band is sharper for the low resistance silicon.

Additional experiments were performed to test the effect of P-type (boron) vs. N-type silicon doping, and to test if there were any changes relative to silicon orientation, $<100>$ vs. $<111>$. No significant differences could be found in data for variations of these parameters. Current choice, N-type and $<100>$ was optimized for easier processing.

Another series of experiment and calculations were performed to ascertain the effect of etch depth of the hole pattern into the silicon. Preliminary data are shown in Figure 13 for both the pattern to detect carbon dioxide and the pattern to detect water vapor. In both cases a very large absorption peak, corresponding to the emission peak, was observed for the deepest etch. The central wavelength of this peak corresponded exactly to the geometrical distance between nearest neighbor holes. In this case the alignment to the geometrical pattern did not significantly alter data. For the holes etched only 0.5 micron deep, the absorption peak was shifted by a factor of about the square root (3/2) and the magnitude was reduced. For the case where holes were only etched in the metal film, the peaks were very small and corresponded to those of the shallow etch in silicon, though shifted in wavelength. 


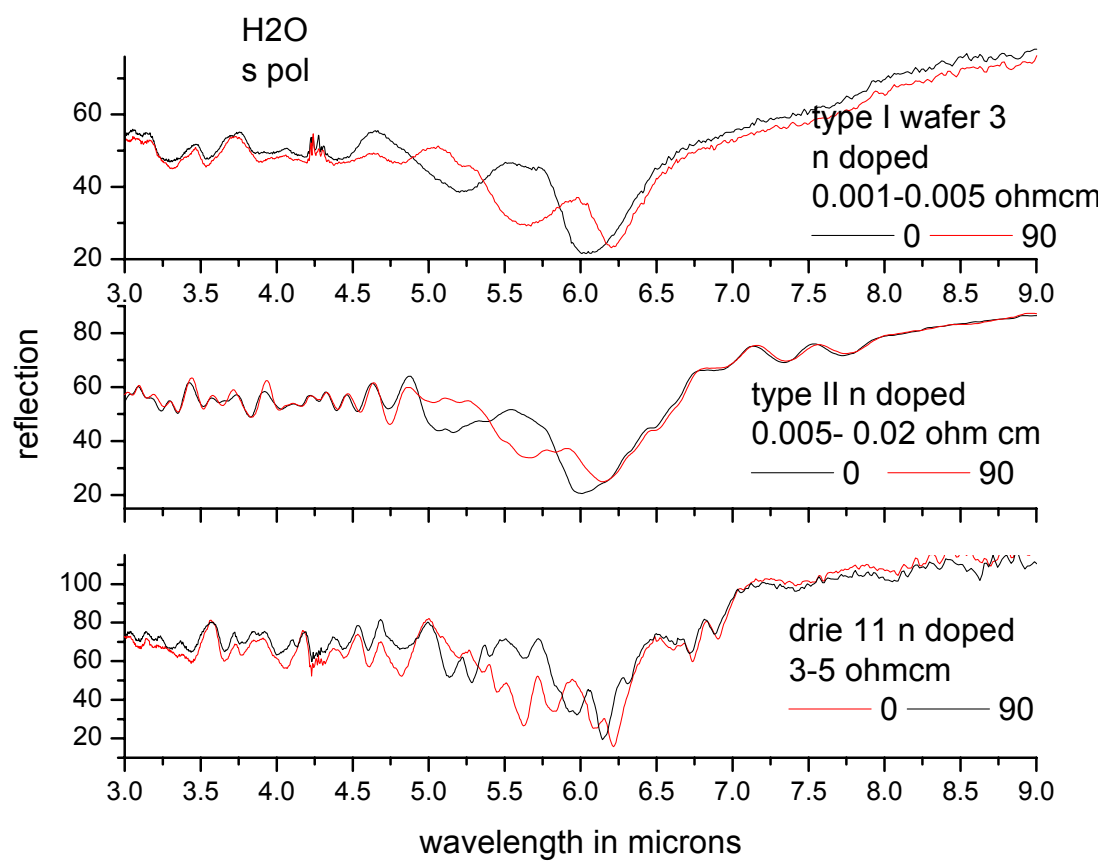

Figure 12 Measured reflection from 2-D photonic crystal used for $\mathrm{H}_{2} \mathrm{O}$ detector as a function of the resistance of supporting silicon.
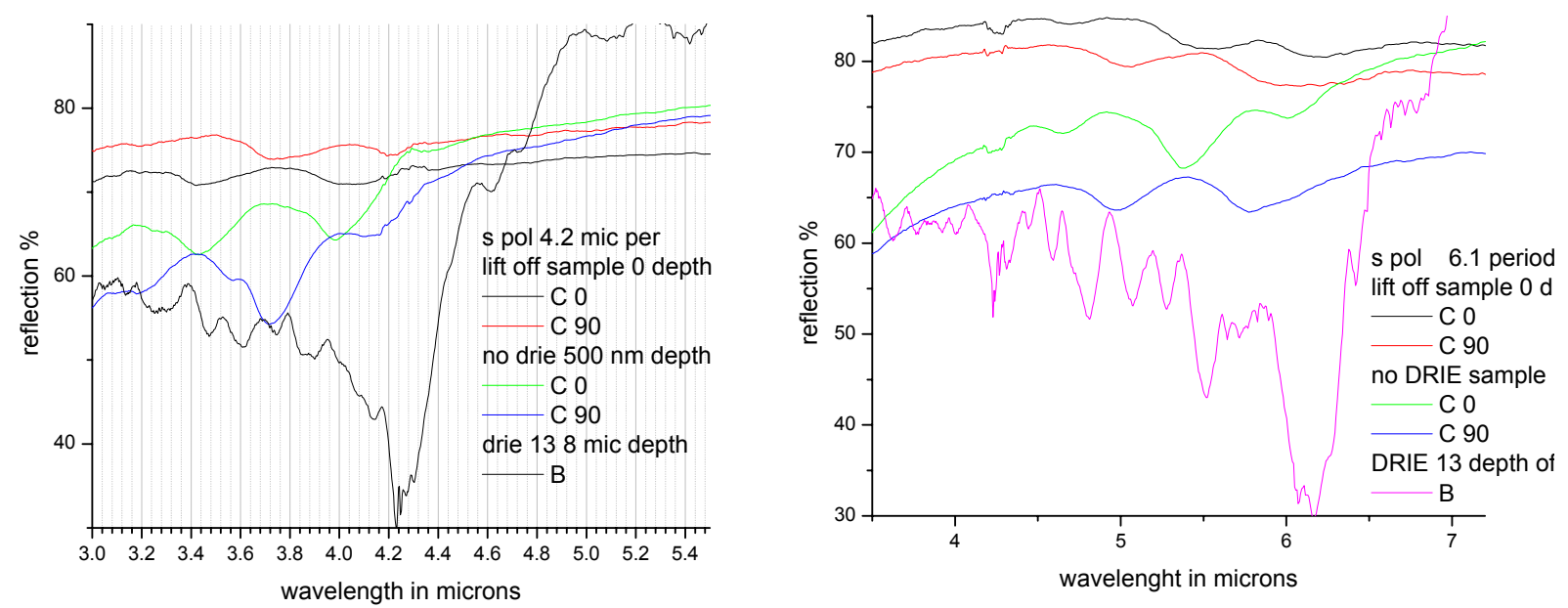

Figure 13 Experimental measurements showing effect of etch depth in silicon on reflection from photonic crystal structure designed for $\mathrm{CO}_{2}$ detection (left) and water vapor detection (right). Black and red lines for zero depth, holes etched only in platinum metal for angle phi 0 and $90^{\circ}$ respectively. Green and blue for holes etched 0.5 micron into silicon for angle phi $=0^{\circ}$ and $90^{\circ}$ respectively. Deep black line (left) or purple line (right) for holes etched 8 microns deep into silicon.

Given the significance of optimization of the depth of etch to maximize the emitted light output in the absorption band of target vapor, which increases signal to noise ratio and sensitivity of the sensor, much time was spent modeling the effect of changes to this parameter and experimental verification. Figure 14 and Figure 15 show model data for $\mathrm{s}$ and $\mathrm{p}$ polarizations respectively for angles $0^{\circ}$ and $90^{\circ}$ to the surface photonic crystal axis. Data are for 4, 6 and 8 micron etch 
depths. The signal is "saturated" for sufficient etch depth. The fine structure of the reflection spectra are not altered, except at very long wavelengths, as a function of etch depth.
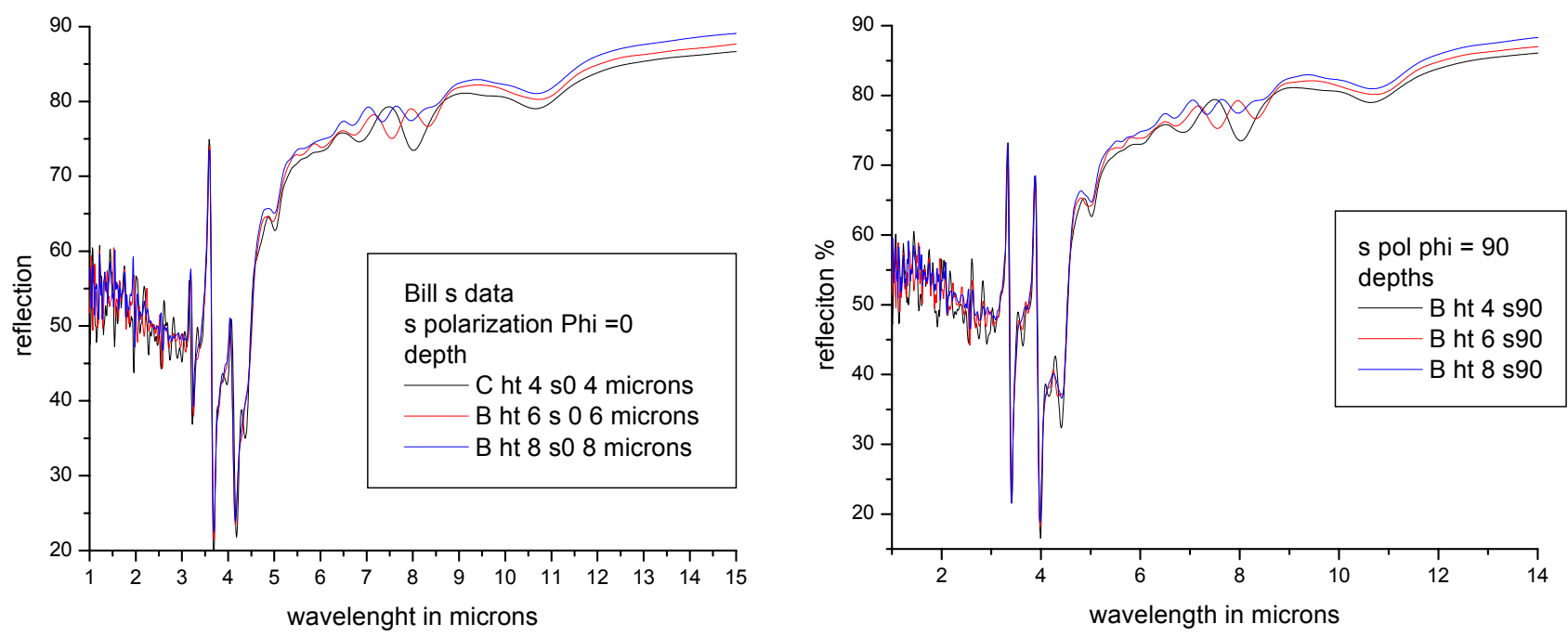

Figure 14 Reflection as a function $f$ wavelength for s-polarization (in plane of incident-reflected light ray) for different depths of etching, 4, 6 and 8 microns deep into silicon. Pattern as shown in Figure 8 .
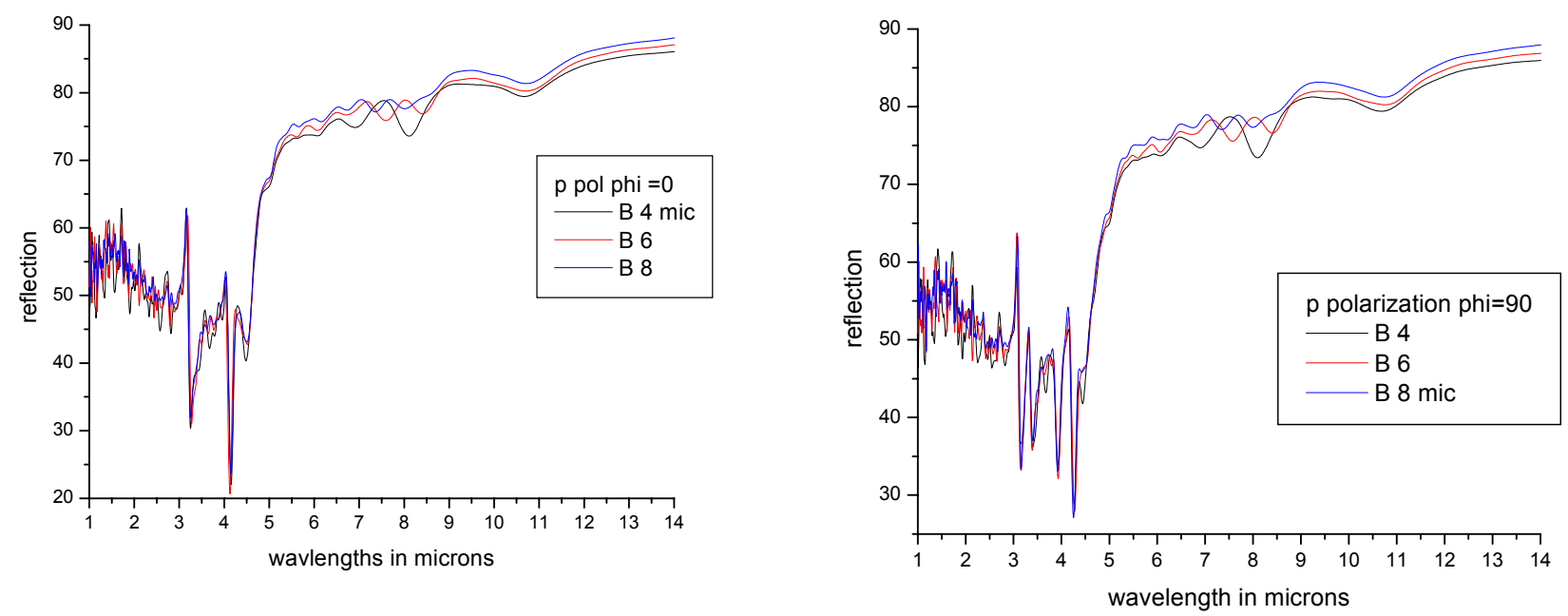

Figure 15 Reflection as a function of wavelength for p-polarization (perpendicular to plane of incident-reflected light ray) for different depths of etching, 4, 6 and 8 microns deep into silicon. Pattern as shown in Figure 8..

The effect of matching modeling results to experimental data is shown in Figure 17. Only the case for 6 microns is shown. We looked at both s- and p-polarization trying to match for angles of 0 and $90^{\circ}$ to the surface pattern. It appeared that the experimental data at $0^{\circ}$ matched model data at $90^{\circ}$. While the modeling data always showed some spectral shifting with this angle, the experimental data always had the resonance at designed wavelength, including separate emission measurements. The modeling data also show larger secondary peaks than experimental measurements. Preliminary explanations for these differences are: 
- The model used wavelength independent index of refraction for silicon. The parameter does vary with wavelength but the computer program cannot account for this now.

- The computer model assumes that the "hole" in the silicon has perfectly smooth walls but deep reactive ion etching (DRIE) leaves scalloped walls, Figure 16.

- Small process changes in material thickness or etch depth do not account for these variations.

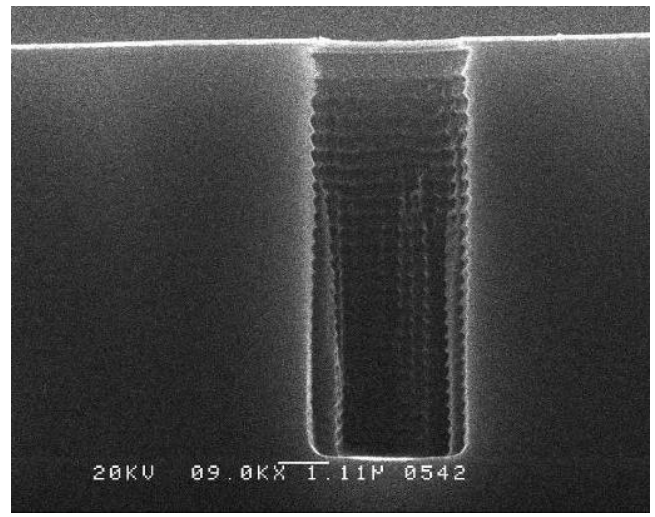

Figure 16 Scanning electron microscope (SEM) photograph of cleaved test sample fabricated for $\mathrm{CO}_{2}$ detection. Depth of etch in silicon is 8 microns, hole diameter at top is 2.1 microns. Walls are vertical but scalloped from DRIE. Bright thin line is metal top layer.
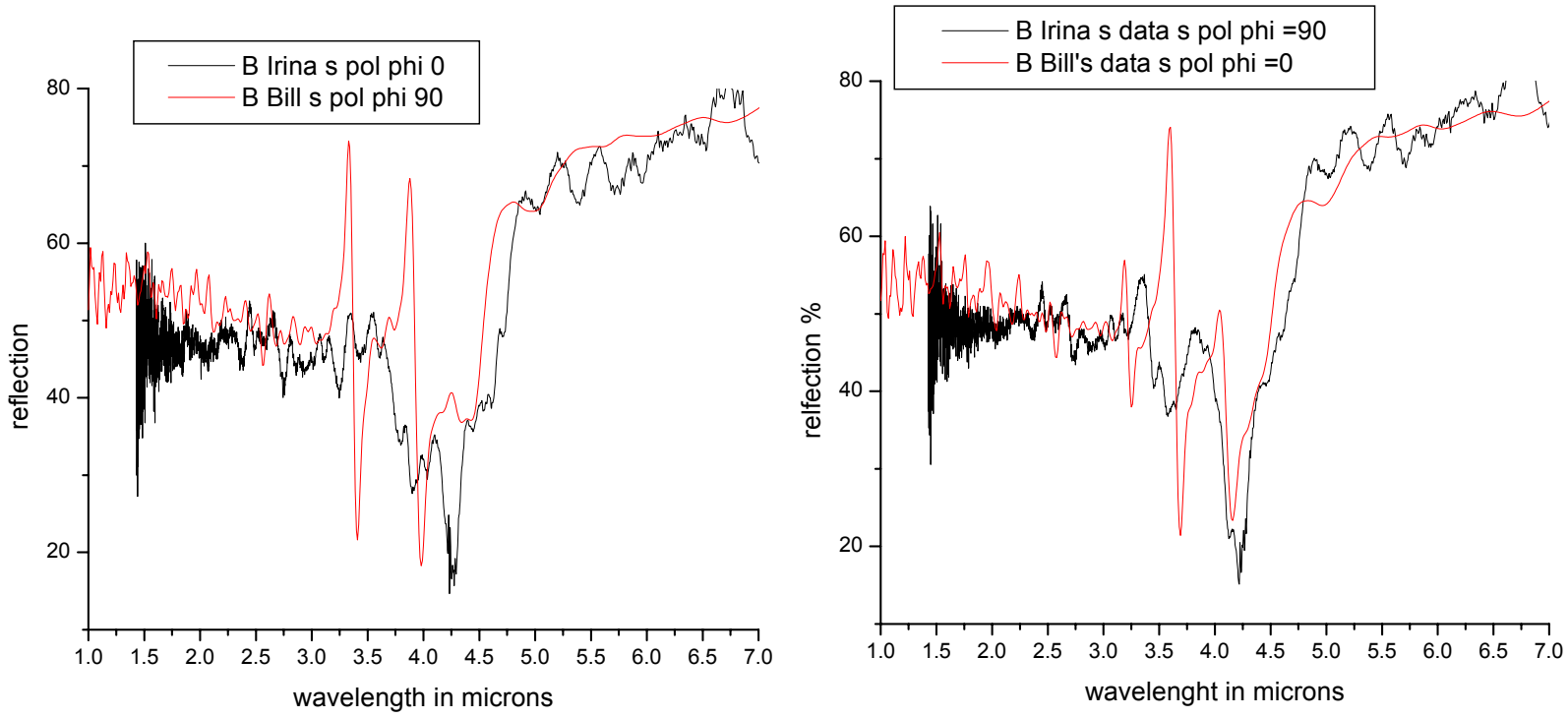

Figure 17 Comparison of theory (red line) and experiment (black line) for 6 micron deep hole in 3-5 ohm-cm silicon for s polarization, reflectance as a function of wavelength, for angle phi at $0^{\circ}$ and $90^{\circ}$. Experimental data shown reversed but always shows peaks at designed wavelength equal to minimum distance between holes (4.2 microns).

Comparison of the computer model and experiment for the case where holes are only etched 0.5 micron into the silicon is shown in Figure 18 (p polarization) and Figure 19 (s polarization). The angle phi in experiment was reversed as in prior case, but the two data sets were taken at $90^{\circ}$ to each other. Peak wavelengths are very well matched. Peak heights differ possibly due to same causes mentioned above. 

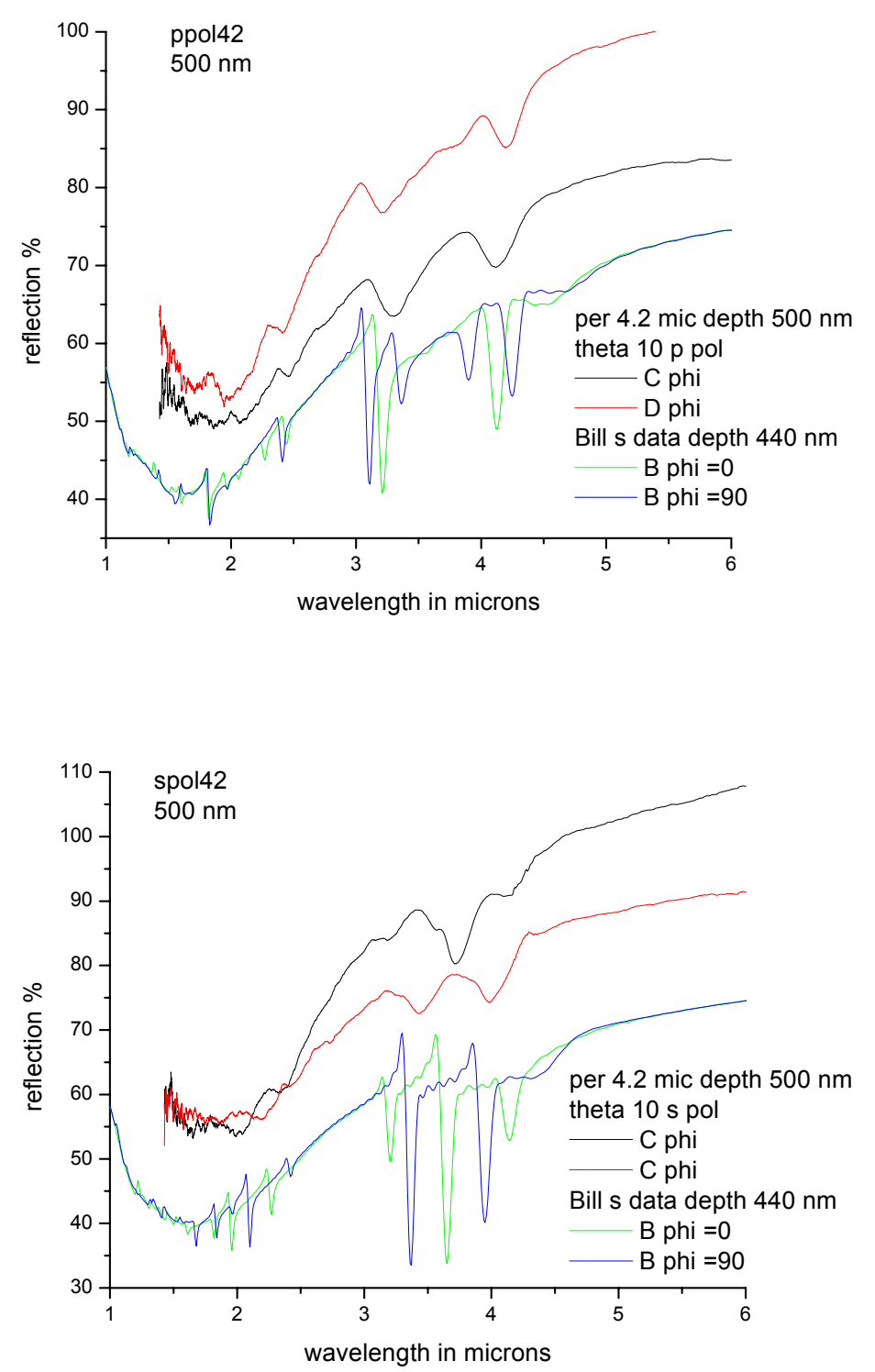

Figure 18 Comparison of theory (blue and green) to experiment (red and black) for hole etched only 500nm into silicon. $P$ polarization. Reflection computed (measured) for angle of incidence of $10^{\circ}$. Central wavelengths are well matched but peak heights are low for experimental case.
Figure 19 Comparison of theory (blue and green) to experiment (red and black) for hole etched only 500nm into silicon. S polarization. Reflection computed (measured) for angle of incidence of $10^{\circ}$. Central wavelengths are well matched but peak heights are low for experimental case.

Additional modeling studies are being conducted by the related NSF funded program with a goal of achieving coherent emission from the photonic crystal. ${ }^{11}$ Results from this other program that could be used for the product developed herein are a study of the effects of hole diameter. Summarizing, the wavelength emitted by the photonic crystal is exactly equal to the minimum spacing between holes for the hexagonal pattern. This central wavelength is not affected by changes in hole diameter.

\section{Thermomechanical Modeling}

The metallization of the MEMS chip was re-designed for this project to reduce parasitic heating and to reduce temporal drift. A major concern was the mechanical strength of the device as it gets very hot (over $350^{\circ} \mathrm{C}$ ) and thermal expansion mismatch between the metal and silicon would create significant strain. This effect was analyzed using finite element thermo-mechanical design programs. 
Ion Optics examined three finite element modeling (FEM) packages: ANSYS Design Space, ${ }^{12}$ FEMLAB $^{13}$ and ALGOR. ${ }^{14}$ In our opinion, the graphic user interface (GUI) for ANSYS was intuitive and much easier to use than either competitor. ANSYS Design Space was purchased by Ion Optics (not charged to this program) with the belief that significant personnel time savings would result for modeling calculations.

The basic geometry of the ANSYS model is shown in Figure 20. We are looking at the MEMS chip and connections (thermal, electrical) to the outside world are included as boundary conditions. The frame is $5 \times 5 \mathrm{~mm}$. The two filaments are about 1.5 by $2.5 \mathrm{~mm}$ area and 10 microns thick (silicon) with appropriate metal films on top. Each filament is supported by two "U" shaped arms with the structural beams being 50 microns wide by 10 microns thick. The stiffness of supporting silicon is sufficient to assure that at rest (no heating) the filaments and structural supports are in a plane with negligible deformation against gravitational force.

Figure 20 shows the part as heated to $350^{\circ} \mathrm{C}$, the computer program amplifying dimensional changes so as to make these visible in the diagram. The metal layers on top of the silicon have greater thermal expansion coefficients than the silicon supporting them and that proscribes direction of bending. The filament goes up and forces the support arm down. As the frame temperature change is minimal, the silicon filament expansion must be accommodated by the "U" support arms being squeezed together. The downward motion of this support and the squeeze from the main filament place the support arm under great stress at maximum temperature. Current room temperature mechanical data for silicon placed in the model indicate that the arms will not fail, but that the generated stress is between 15 and $50 \%$ the failure point of the material. At high temperatures the plasticity of silicon and the safety factor in this design may be greater than this indication. The fact that our device did not fail after very many test cycles implies this is true. Limited data availability (mechanical properties of hot single crystal silicon) limits application of computer modeling.

The model gave insight into thermal loads. If the device is not well sealed and thermal convection is appreciable (and much greater than radiation), then the electric current heating the support arms drives that part to a higher temperature than the filament and heat flow is backwards, from the arms to the filament, Figure 21. In this case, substantial thermal flux is incident on the device window not visible in this calculation. If the package is well sealed and thermal convection minimized, then heat flow is in the expected direction, from the filament through the arms to the frame, Figure 22. Reducing power drawn by the device requires good vacuum sealing. 


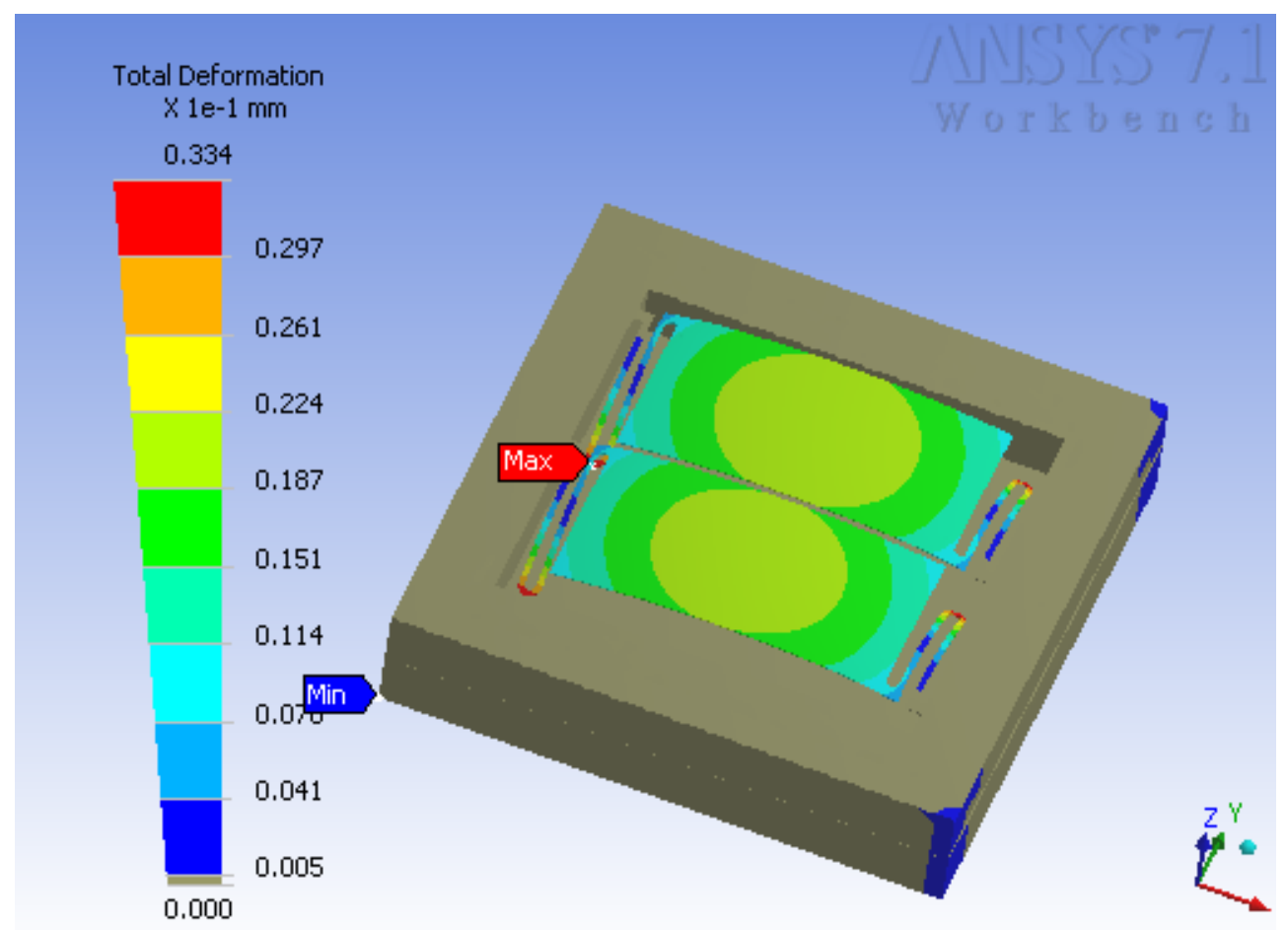

Figure 20 Mechanical distortion of heated MEMS device. At a peak temperature of $350^{\circ} \mathrm{C}$ the support arms bend downward by over 33 microns, this is about $3 \%$ of their overall length of 1500 microns.

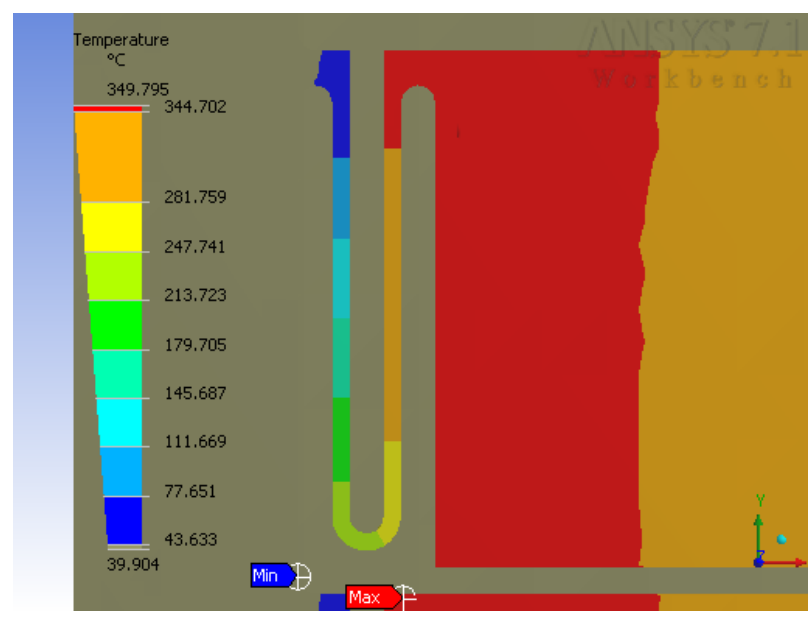

Figure 21 Thermal profile of MEMS chip with convection losses equivalent to that at a pressure of 100mtorr showing reverse heat flux from " $U$ " shaped support arms to the filament.

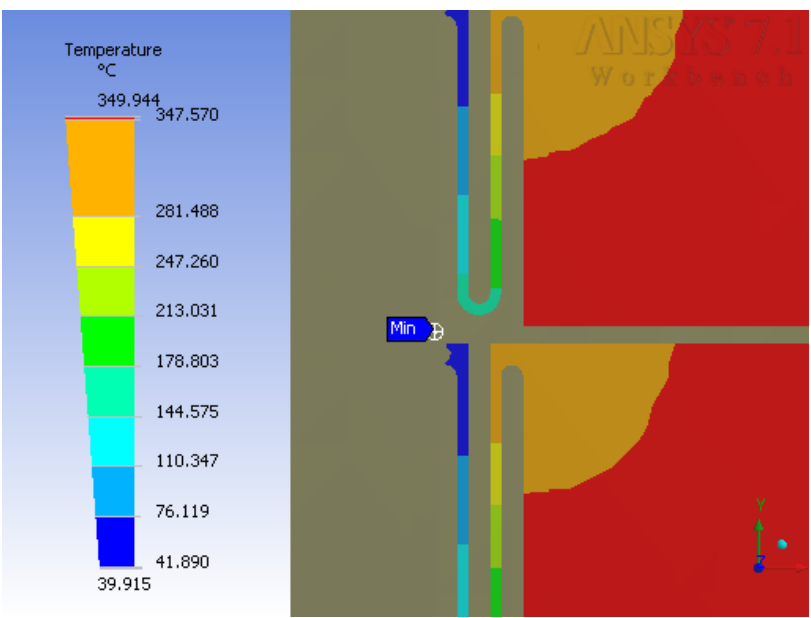

Figure 22 Thermal profile of MEMS chip with convection losses equivalent to that at a pressure below 10mtorr showing heat flux from the filament to support arms. 


\section{Optimization of Diffusion Barrier}

Between the silicon supporting membrane, nominally 10 microns thick, and the top layer of metal necessary for photonic crystal function is a diffusion barrier. Experiments conducted prior to the start of this contract tested silicon oxide and silicon nitride for this function. Either can function as such a barrier but, for compatibility with the MEMS fabrication process, $\mathrm{SiO}_{2}$ was chosen. These early experiments indicated that emission intensity was reduced as the thickness of this layer was increased. The minimum thickness is that which guarantees a continuous layer after deposition, about 50nm. The MEMS process sequence, however, required thicker $\mathrm{SiO}_{2}$ to maintain layer integrity when etching the metal layers deposited on top. The (proprietary) thickness now used is compatible with requirements of the particular fabrication house chosen for manufacturing the MEMS device, and is identical to that in our computer model.

\section{Optimization of Metallization}

At the high temperatures maintained in the emitter, metal layers will diffuse into and react with other metals forming alloys. This alters electrical properties and causes the sensor to "drift" or change calibration. As a sensor installed in a building is expected to have a very long useful lifetime, this drift must be zeroed. The problem was addressed by testing different thicknesses and materials for conductors and diffusion barriers. A combination was identified that, with stabilizing post-processing thermal anneal, reduced drift by several orders of magnitude.

Thermal expansion mismatch between the $\mathrm{SiO}_{2}$ diffusion barrier, the silicon support and the top metal layer could lead to delamination with repeated thermal cycling between room temperature and $350^{\circ} \mathrm{C}$. Adherence promoting layers between the $\mathrm{Pt}$ and silicon-dioxide layer were necessary. Titanium, tantalum and chromium were tested with titanium preferred. Tungsten and Nickel were tested for use as bus bars - the layer that must carry full current to the heater along the support arm at high current density. For the layer that must act as the top of the photonic crystal, gold, and platinum were tested.

Tests of different metals for the top layer of the photonic crystal were completed at Ion Optics' expense during the interim period between writing of the proposal and start of contract. Early tests with use of gold were performed in the 1999 to 2001 time frame. Gold, originally chosen for high IR reflectance, high conductivity, and low chemical interaction proved to react with silicon and silicides at $350^{\circ} \mathrm{C}$. The long term stability of gold was not good. Platinum is now the metal used for the photonic crystal.

Is there an optimum thickness for the top metal layer? Two effects are considered - emission from the photonic crystal and resistance value and stability for electric heating. OEM customers indicated their desire to use low voltages (preferably under 3 volts) for driving sensors to prevent their being a potential ignition source in the unlikely event of high concentration of volatile vapors, such as a natural gas leak. The serpentine pattern of the top metal layer visible in Figure 5 has five legs (zigzags) and a resistance of about $100 \mathrm{ohms}$ for a 100nm layer of platinum. Uniform heating would be difficult to control on a thinner layer. An increase in thickness requires additional legs to maintain identical total resistance and that would reduce area devoted to metal. However, this can be managed for a change of about a factor of two. A test of increasing metal thickness was performed, Figure 23. At $250 \mathrm{~nm}$ little difference in emission was detected (a slight increase in power emitted is attributed to a slight increase in hole size). To 
improve yield and relax processing restrictions, 100nm thickness of Pt with $10 \mathrm{~nm}$ of adherent promoting metal underneath was adopted.

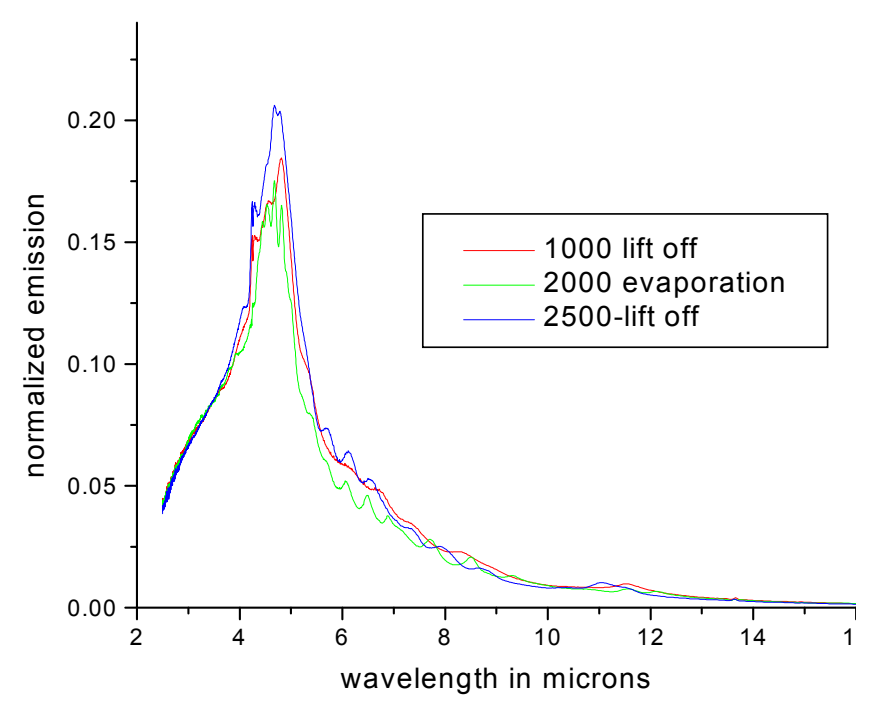

Figure 23 Emission from photonic crystals with varying thickness (1000, 2000 and $2500 \AA$ ) of Platinum. Variations were traced to lithography defects.

Studies to optimize the adherence promoting layer were performed next. Pure platinum films did not adhere to the MEMS device. Thermal annealing after deposition was not possible as these films peeled off almost immediately after deposition. Routinely, following published practice, thin layer of titanium or tantalum are used to promote adhesion between platinum and silicon or silicon dioxide. A chemical reaction with $\mathrm{SiO}_{2}$, the formation of an atomic layer of metal-oxide, promotes adhesion. Heating these as-deposited films (10nm Ti or Ta, $100 \mathrm{~nm} \mathrm{Pt})$ continuously at operating temperatures $\left(320^{\circ} \mathrm{C}\right)$ caused Ti-Pt or Ta-Pt reactions. The rate of change of metal resistance measured at high temperature was $1 \%$ per day for $\mathrm{Ti}$ and $4.6 \%$ per day for Ta.

We performed an experiment to determine if these films could be stabilized by thermal annealing immediately after deposition at temperatures greater than the operating temperature. Preliminary data from another DOE program had indicated that Ti-Pt contacts could be stabilized. ${ }^{15}$ In this project, a matrix experiment was performed for both $\mathrm{Ti}$ and Ta testing different temperature-time cycles. The best result is shown in Figure 24. A summary of the total experimental matrix is shown in Figure 25. The two filaments were held at a temperature of $320^{\circ} \mathrm{C}$ for two days and the current-voltage curves plotted as resistance versus time. Line thickness is caused by noise and would be much thinner if we only plotted a 30 second average value. The high temperature anneal reduced drift to less than $0.05 \%$ for Ta and $-0.017 \%$ for Ti. The latter figure is just within the resolution of the experiment and the absolute value of the drift may be lower. For comparison, we placed a platinum RTD in an oven at $320^{\circ} \mathrm{C}$ and measured the drift of this commercial device renowned for its stability. The drift of the RTD was comparable to that of the $\mathrm{CO}_{2}$ sensor developed in this project. 

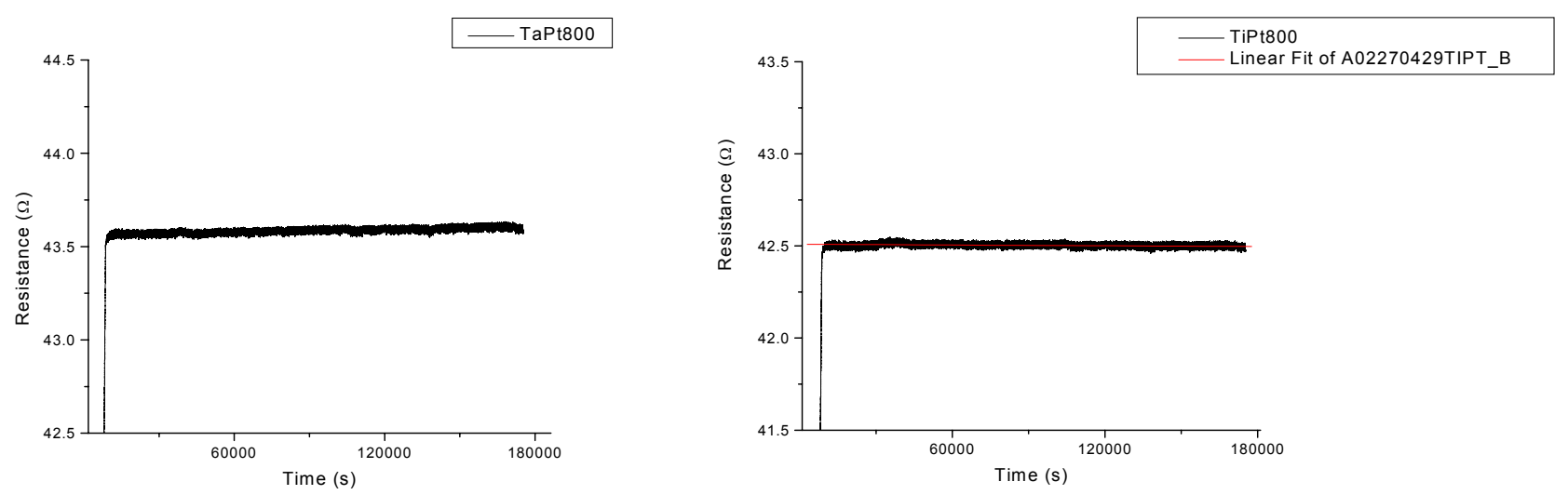

Figure 24 Stability of high temperature annealed TaPt (left) and TiPt (right) measured at 320C. Temporal stability of TaPt $=0.047 \% /$ day. Temporal stability of TiPt $=-0.017 \% /$ day.

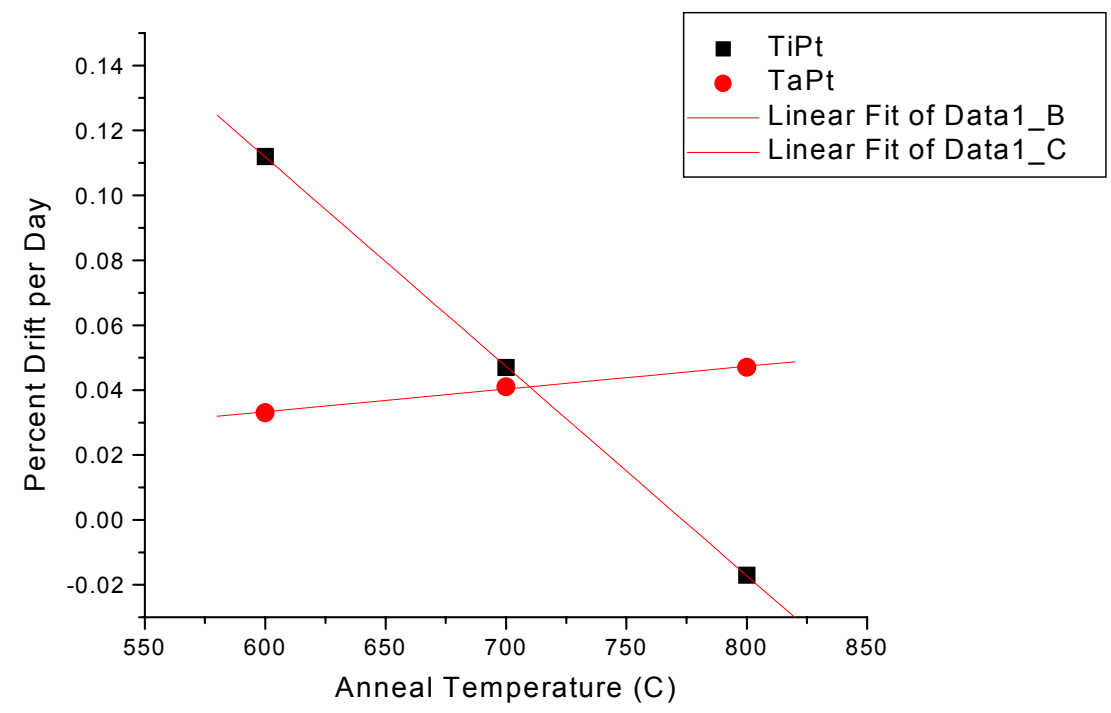

Figure 25 Drift per day (Figure 23) in percent as a function of temperature of anneal for TiPt and Ta-Pt metal layers. Film thickness was 10nm of Ti/Ta and 100nm for Pt.

A transmission electron microscope (TEM) study was performed to determine the science behind this result and determine if it could be further improved. In Figure 26 the as-deposited titanium film (a) is of uniform thickness and continuous. After annealing at high temperature the titanium is repositioned and is concentrated in large, spherical grains at the center of the film thickness. These grains are evenly spaced, Figure 27 . There is some small amount of titanium at the original interface, but this remainder is not continuous. 

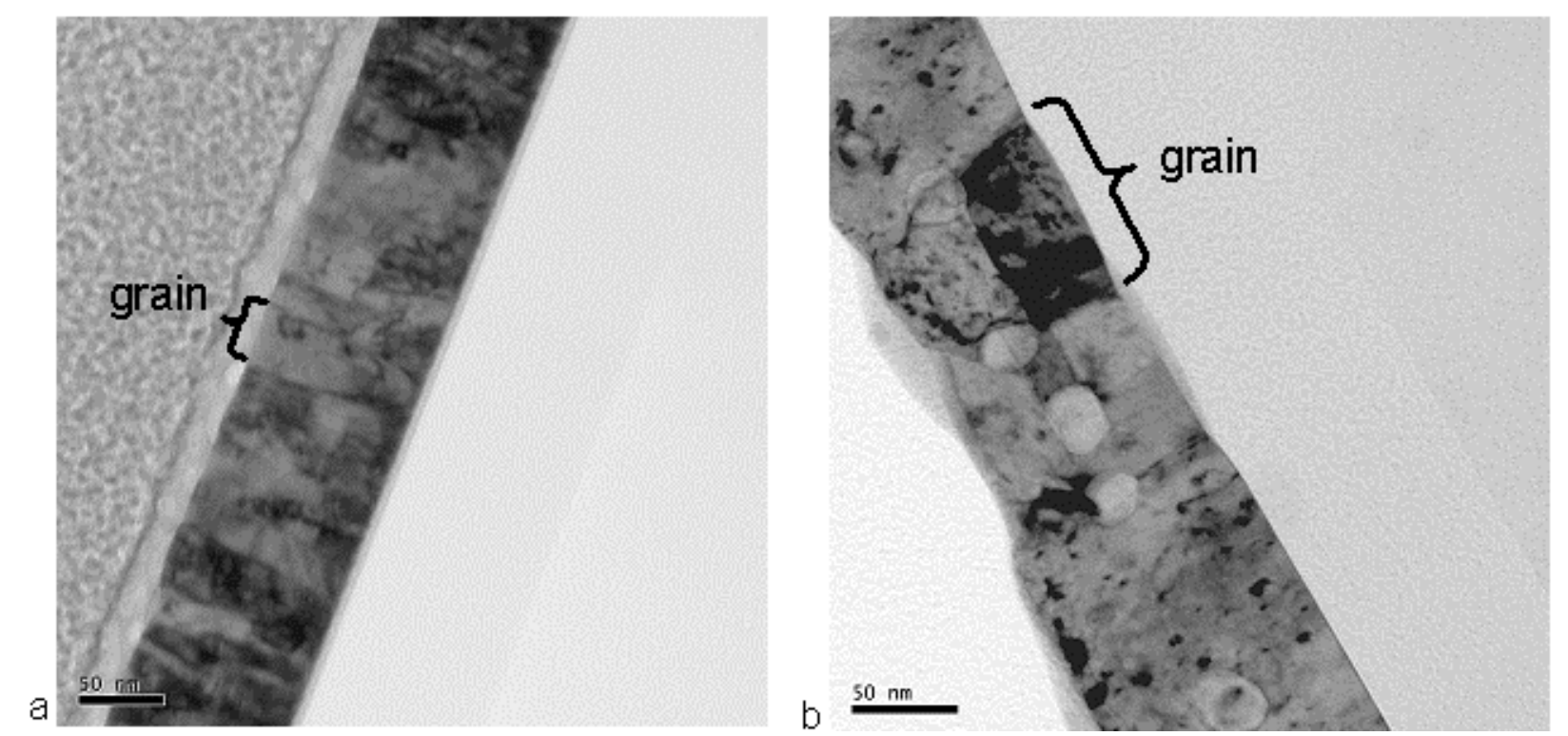

Figure 26 Micrograph before (a) and after (b) annealing at $800^{\circ} \mathrm{C}$. In micrograph (a) the titanium is uniformly layered continuously between the platinum and the oxide while in (b) the titanium is phase separated into discrete spherical shaped grains.

After annealing, the adherence of the Pt film is good. We believe that a very thin platinumsilicide layer is formed at this high annealing temperature and that stabilizes the $\mathrm{SiO}_{2}-\mathrm{Pt}$ boundary. The thickness of this layer did not seem to change with time, but very long term stability studies are still in progress.

In contrast to the Ti-Pt system, the Ta-Pt metal system showed little or no diffusion of the adherent layer (Ta) after high temperature anneal (Figure 28). This system appears to be more chemically stable than the Ti-Pt system, but because it shows some change in resistance (drift) with time at $350^{\circ} \mathrm{C}$, the Ti-Pt metallization was chosen for prototype fabrication.

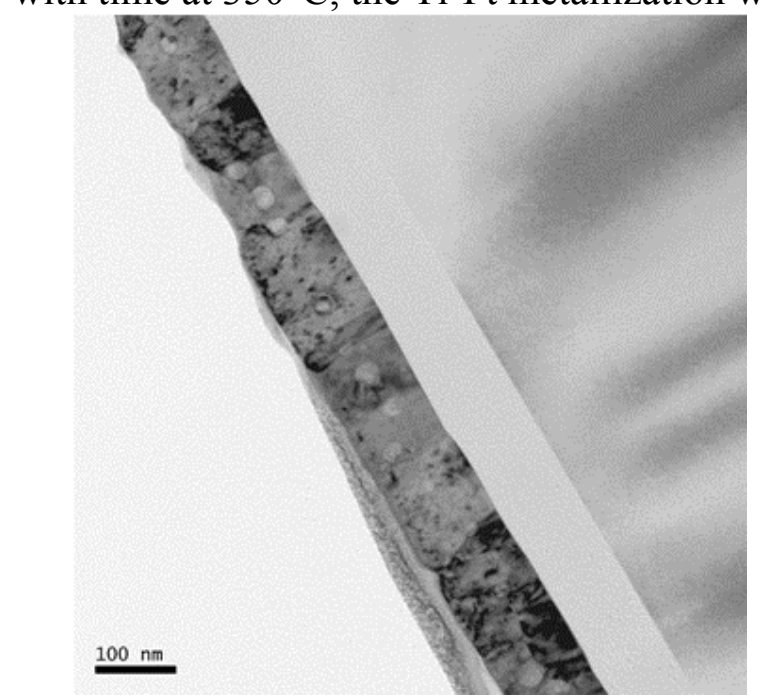

Figure 27 Zoomed out view of the annealed Ti-Pt film showing uniformly sized titanium rich grains in the platinum bulk. The grains are uniformly spaced, suggesting a diffusion limited process.

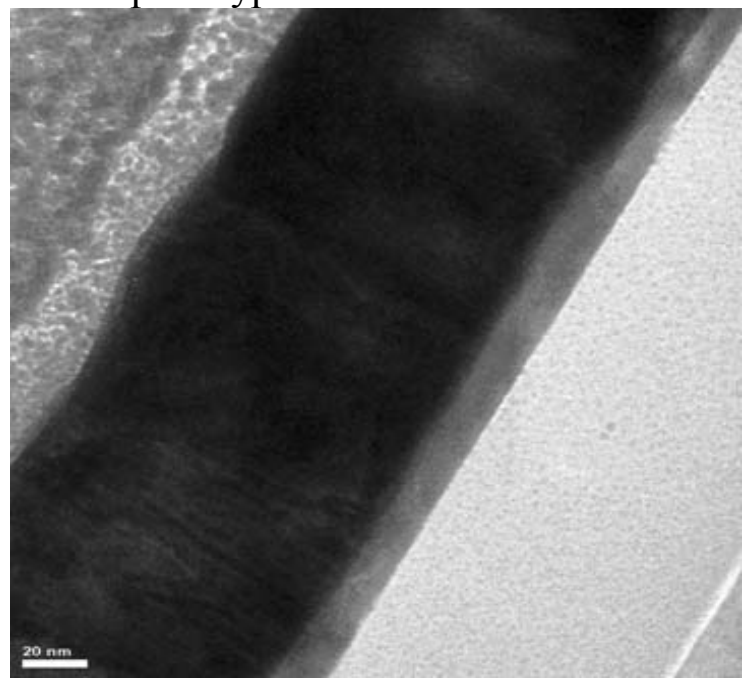

Figure 28 Cross sectional TEM micrograph of an annealed Ta-Pt metallization. From bottom right to top left: silicon, silicon dioxide, tantalum, platinum, preparation medium. 
The last metal layer to be optimized is the bus bar, the layer carrying current over the U-shaped support arm to the filament (see Figure 21). The extra thick metal layer is deposited on top of the platinum layer after high-temperature thermal annealing. Large interdiffusion of these layers is avoided, but alloying does occur during operation at $350^{\circ} \mathrm{C}$. To remove this bus-bar change from the measurement, a four-wire connection is used to measure the voltage across the platinum filament heater. A wide metal line is used to carry the high current while a smaller metal line is used to carry the (very low current) signal. A microscope image of these wires is shown is Figure 29. The metal layers are deposited on top of the diffusion barrier on the silicon support. This barrier also acts as an insulator preventing electrical connections between the two lines, and between these lines and the silicon support. Connections through the silicon would short out the electrical device.

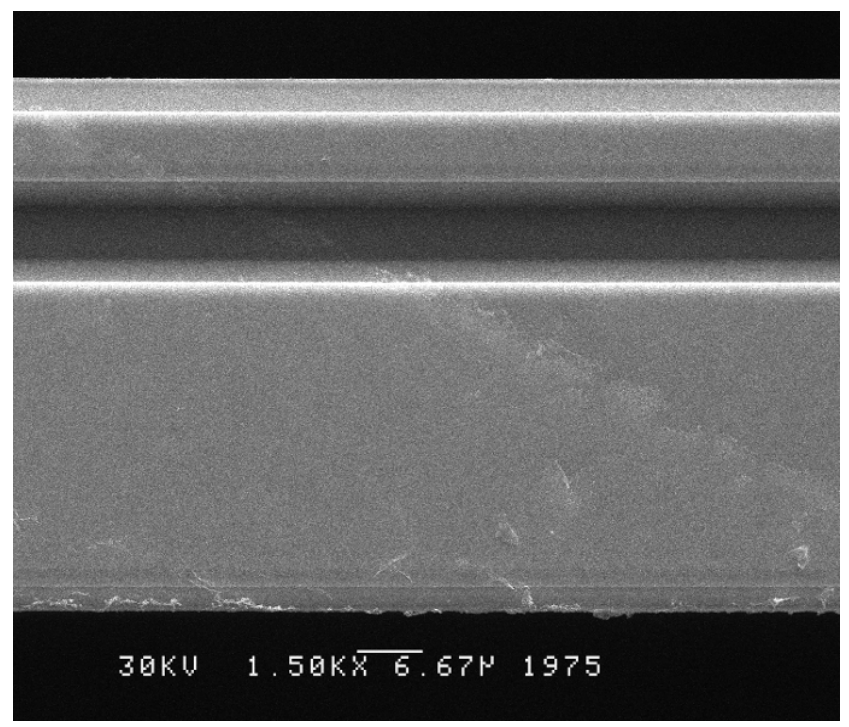

Figure 29 Scanning electron microscope image of bus-bar on support arm. Total width of support arm (top to bottom in picture) is 50 microns. Designed dimensions for wide metal line is 30 microns, thin line is nominally 5 microns, space between lines and between the line and edge of support is 5 microns.

The current carrying element of the platinum heater which also acts as the top layer of the photonic crystal is about 0.1 micron thick by 300 microns wide. The bus-bar is about one micron thick but only 30 microns wide. Excepting that the effective cross section of the platinum layer is reduced by the photonic crystal pattern, the current densities in the heater and in the bus-bar are equal. The metal layer for the bus-bar should have a low electrical resistance to avoid overheating and must be compatible with high temperatures. Making this layer thicker would be preferable but is not possible.

For this application, the following evaporated metal systems have been tested:

Ti-Pt-Ti-Ni

Ti-Pt-Cr-Ni

Ti-Pt-Vent the chamber-Ti-Ni

Ti-Pt-change to sputtering-Ti-Ni

Ti-Pt-Ti-change to sputtering-Ti-Ni

Ti-Pt-Ti-change to sputtering-TiW-Ni

TiW-Pt-TiW (sputtered)

In this initial experiment, all metal stacks survived the $350^{\circ} \mathrm{C}$ annealing temperature. The second important observation from these experiments is that samples that were vented in middle of the deposition were no different than similar samples that were deposited in one continuous process. 
Furthermore, there was no difference between sputtered and e-beam evaporated samples. These observations allow broad process flexibility for device fabrication. In a second experiment, the following metal stacks were further processed to yield patterned electrical test structures:

Ti-Pt-Ti-Ni

TiW-Pt-Ti/W (sputtered)

Ti-Pt-Ti-Ni-Cr

Ti-Pt-Ti-Ni-Au

The first two of these successfully survived the patterned step but the latter two did not pattern very well. Samples were wire bonded into chip carriers but not sealed. They were tested in a high temperature vacuum chamber. Results are summarized in Table 1.

Table $1 \quad$ Resistance variation with time at $350^{\circ} \mathrm{C}$

\begin{tabular}{|l|l|l|}
\hline Materials & Drift (ohm/sec) & Normal. Drift (\%/day) \\
\hline Ti-Pt-Cr-Ni-Al (Spin5) & $6.48 \mathrm{e}-6$ & 8 \\
\hline Ti-Pt-Ti-Ni & $3.3 \mathrm{e}-6$ & 0.4 \\
\hline Ti/W-Pt-Ti/W & $2.02 \mathrm{e}-6$ & 0.42 \\
\hline Ti-Pt-Ti-Ni-Cr & $3.2 \mathrm{e}-6$ & 2.5 \\
\hline Ti-Pt-Ti-Ni @ 250C) & $3.5 \mathrm{e}-7$ & 0.056 \\
\hline
\end{tabular}

From the data table it is quite clear that $\mathrm{Ni}$ and $\mathrm{Ti} / \mathrm{W}$ are the two candidate metallizations from a purely drift perspective. Other considerations are important - such as the ability to pattern the metal stack and its high temperature resistance. The resistance of $\mathrm{Ti} / \mathrm{W}$ alloys was nominally $7.5 \mathrm{e}-5 \mathrm{ohm}-\mathrm{cm}$, compared to a resistance of $5 \mathrm{e}-6 \mathrm{ohm}-\mathrm{cm}$ for pure tungsten. This effectively eliminated the $\mathrm{Ti} / \mathrm{W}$ metallization from contention since the absolute arm resistance would be too high and comparable to that of the heater. The Ti-Pt-Ti-Ni metallization became the metal stack of choice for the bus-bar. At slightly lower temperatures, this metal stack yields additional order of magnitude stability.

\section{Electronics}

All of the experiments described to this point were conducted with large laboratory instruments to collect data. The sensor was driven by high precision, programmed power supplies (from Kiethley Instruments) and readout performed by a data acquisition card (from National Instruments) inserted into a PC computer. The program LABVIEW ${ }^{\mathrm{TM}}$ (National Instruments) was used to input and analyze digitized data. To operate the sensor as a commercial product, it must be driven by a low parts count circuit with a single digital output. The design problem is to do this while maximizing sensitivity and minimizing drift.

The signal from the sensor is a change in temperature of the filament due to varied absorption of light when the target vapor absorbs some of the radiated energy (Figure 1). The change in temperature causes a change in material resistance and the resulting variation in either voltage or current is measured. This is a small change in a large signal and it is difficult to obtain high precision. If we use a second filament as a reference not varying with target vapor concentration, then the two voltages can be subtracted so that the true signal of gas concentration is a large change on a small background and precision is improved. This is the principle of the Wheatstone bridge circuit (Figure 30). 


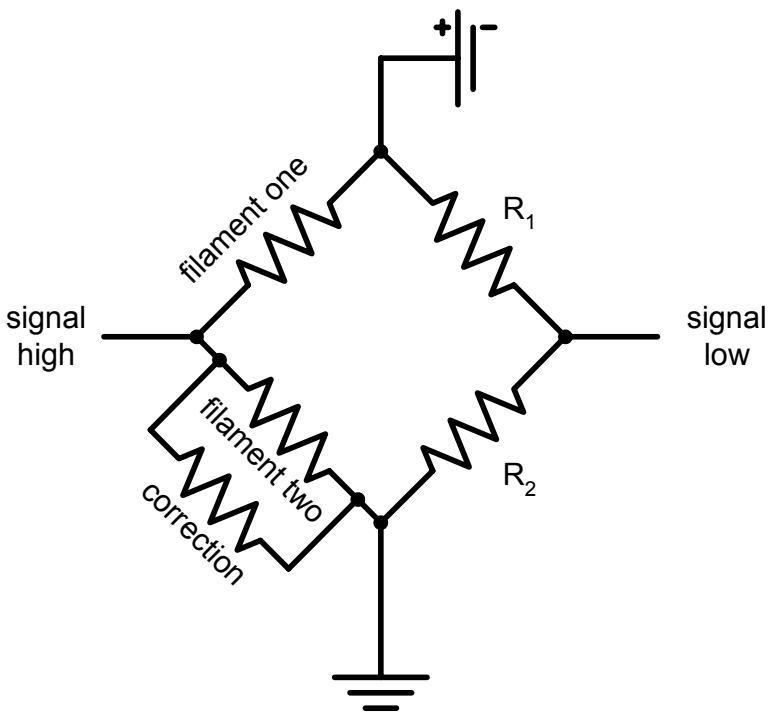

Figure 30 Wheatstone bridge circuit for drive and readout of gas sensor. Filament one and filament two are on one MEMS chip. One is covered by optical block on window of package. Correction resistor adjusts total resistance so that both filaments are at identical operating temperature. $R_{1}$ and $R_{2}$ are matched precision resistors.

In the absence of drift, as discussed in previous section, this circuit has very high precision. Only one filament has an optical signal that varies with vapor concentration. In the absence of absorbing vapor, nominal potential difference between the filaments is nearly zero - there is a small change due to different optical reflection from the mirror in the gas cell and the mirror blocking the reference. The change in this signal due to gas absorption is larger so that the signal to noise ratio is improved and the precision of the sensor is good. This circuit is the test device that is being distributed to potential customers of Ion Optics at the end of this project (Figure 31). It is very small, requires little power, and can meet specifications for most anticipated applications. Note that the total sensor volume with mirror is less than the target $100 \mathrm{~cm}^{3}$.

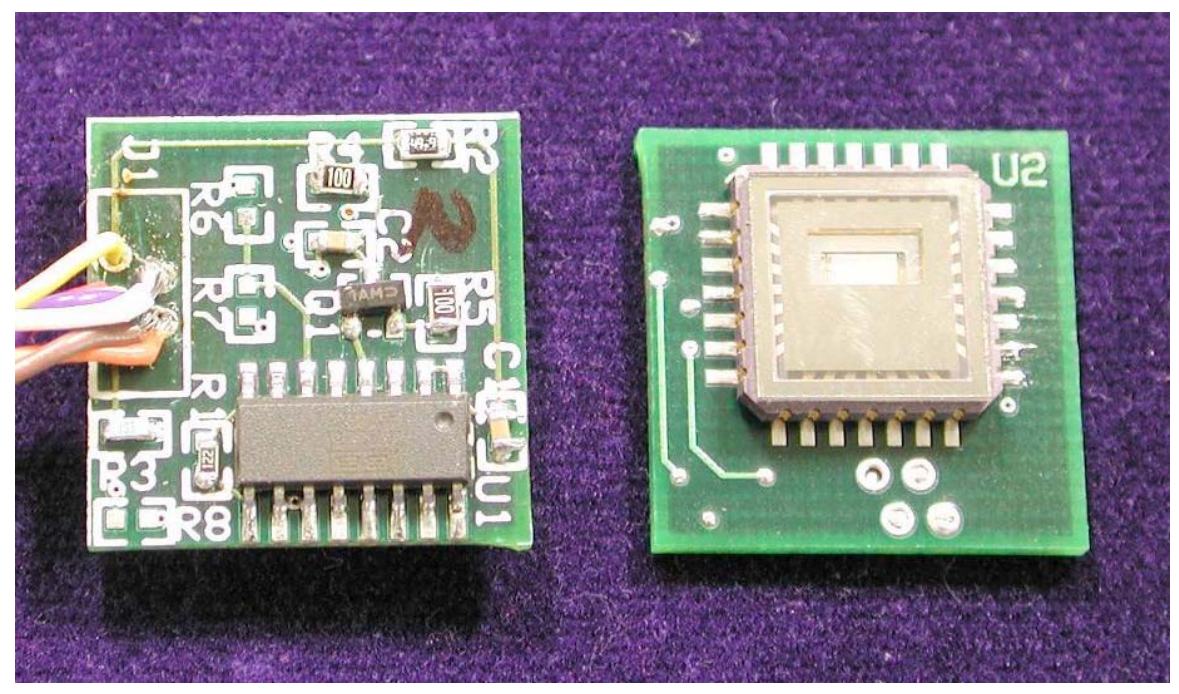

Figure $31 \quad$ Prototype $\mathrm{CO}_{2}$ sensor, packaged in LCC. Total size is about one square inch and a few millimeters height. The chip, on the right, shows that one pixel is "blinded" (compare to (Figure 5) and used for temperature compensation. The circuit board on the reverse of the chip (left) has one IC and a few resistors (Figure 30). 
In practice, there is drift of the filament resistance, especially in the support arm leads. As this circuit forces the current in each filament to be identical, the filament with slightly higher resistance after drift would go to a slightly higher temperature. That change increases the rate of drift in the filament with higher resistance further increasing the difference between the two parts. The total effect is very small, but after many years signal drift could be large enough to prevent correction with periodic recalibration.

With the four-wire circuit, the signal would be determined separately for each filament. The signal is no longer determined by the potential of the driving circuit but by the potential of the sensor. The two signals can be added (subtracted) in an analog circuit preserving precision. However, if the Wheatstone bridge is still used the current through both filaments cannot be individually adjusted so that the drift with time also becomes unstable eventually. This may take a long time, but we are aiming for a device to be used for building controls with an expected lifetime of about 20 years so stability had to be improved.

For maximum stability during our extended testing cycle, using the four wire circuit on the sensor chip to maximum advantage, each filament is powered separately (Figure 32). The potential across the sense wire is used as a control signal as well as the output signal. A digital signal processor (DSP) controls the input voltage to each sensor. The processor is preprogrammed to drive each filament to a given resistance (temperature). The very small rate of drift in the platinum heater can be preprogrammed into the DSP memory. The output across the sense wires passes through an OP amp (operational amplifier). This signal can be split with part going to the DSP and part going to analog subtraction from the other signal (option shown). A change in target vapor concentration would alter the drive to maintain constant temperature and this becomes the sensor output. 


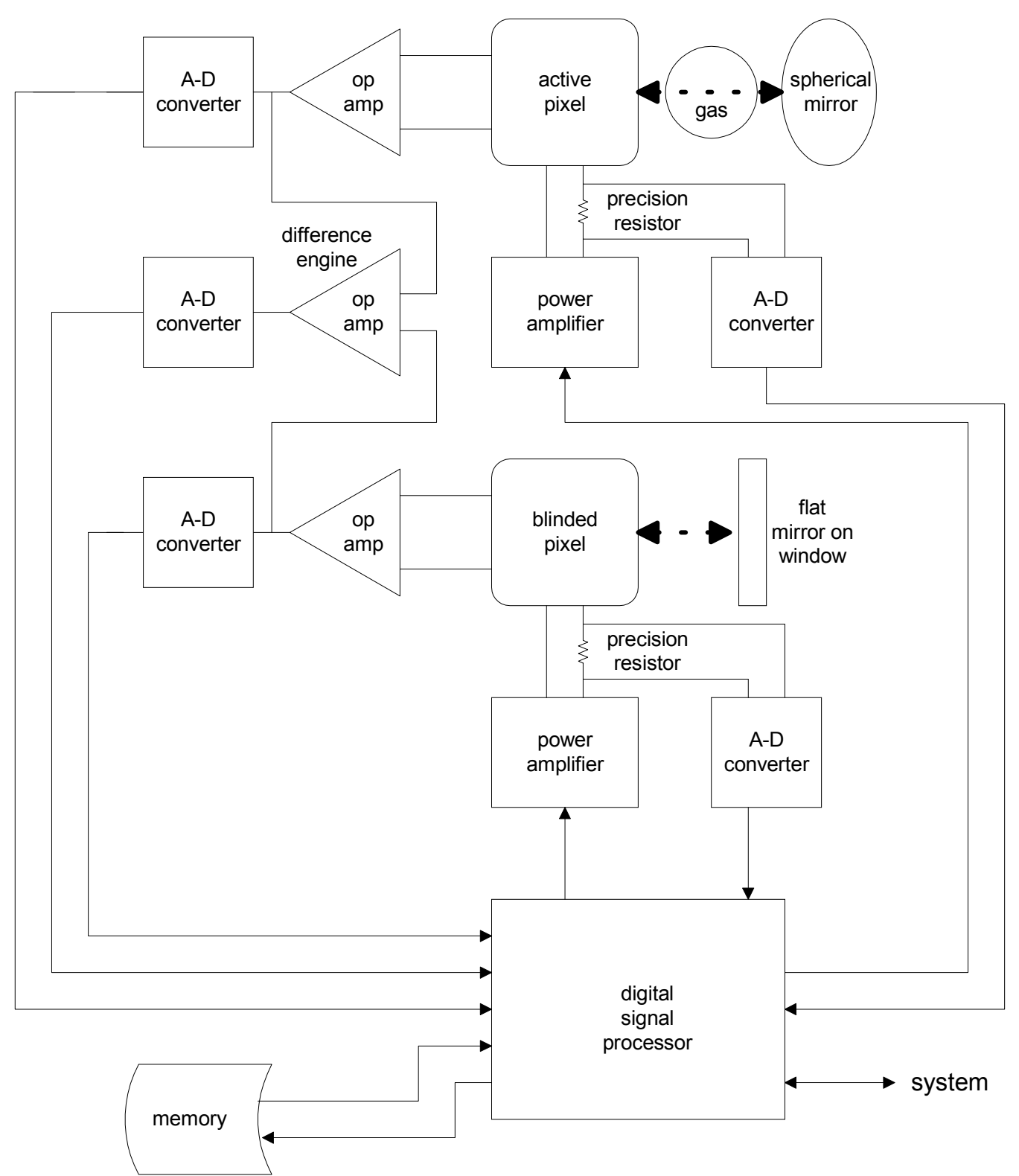

Figure 32 Schematic diagram of optimal control scheme using 12 IC's. Total can be reduced to seven with multiplexing, use of dual op-amps. Circuits are small, common and inexpensive.

Because this circuit uses only the potential across the platinum resistor for control, and because the drive current though each filament is independently controlled, the drift of the support arm resistance is completely removed from the problem. Large changes over a very long period of time can be accommodated without altering the precision output. Because every effort is aimed at maintaining equal temperature of both filaments, long term drift of each should be nearly equal. What happens if there is a change in room temperature? Both filaments are mounted close together on the same frame and respond in an identical fashion to environmental insults. Subtraction of signals cancels all outside temperature effects. Advantages of this control circuit in measured drift rates of the sensor are presented in Figure 33 and Figure 34. Drift with room temperature has been effectively zeroed out. Drift with time has been reduced to a level about equal to electronic noise. 

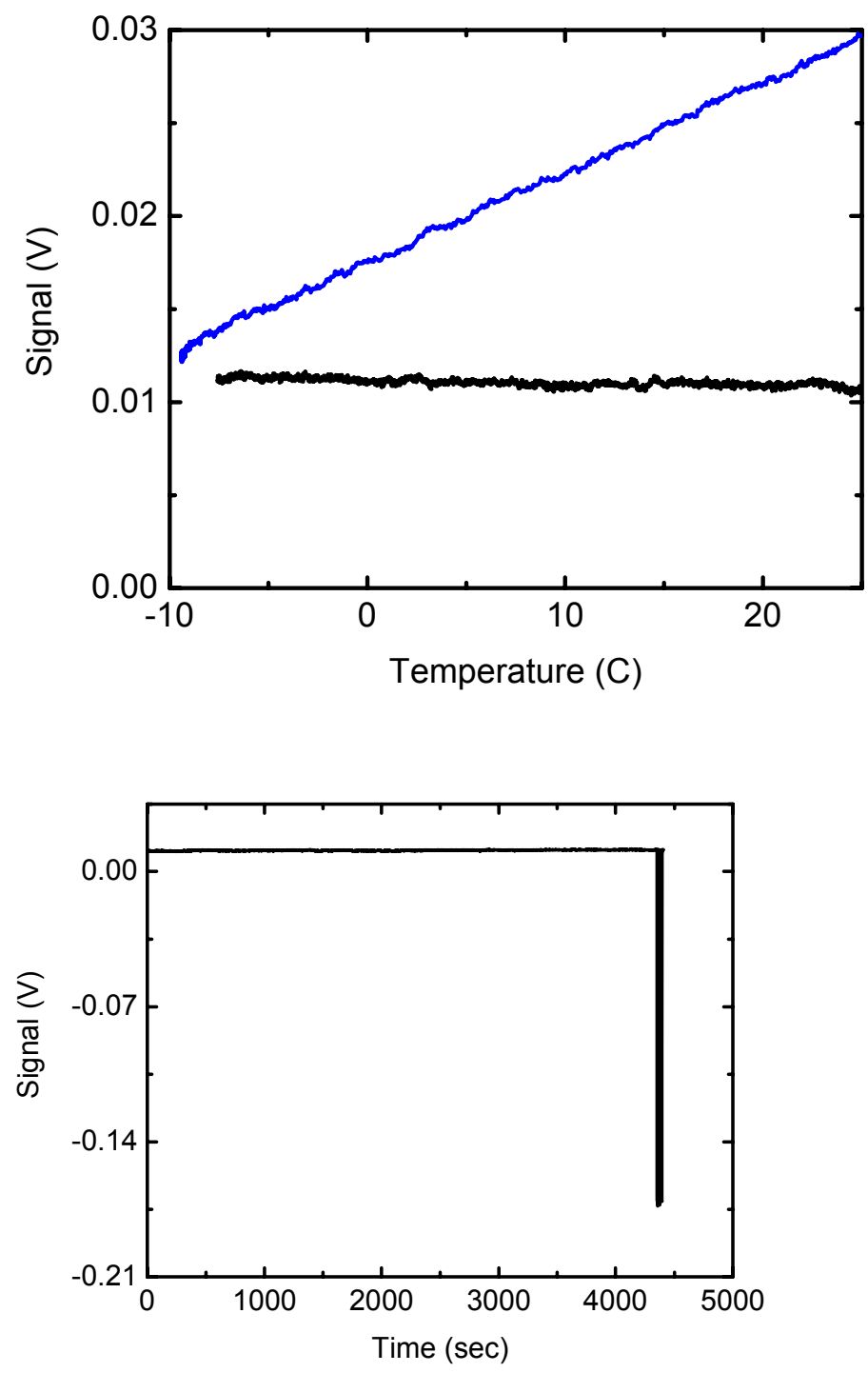

Figure 33 Drift of baseline signal with room temperature as originally measured at start of this project (blue line, angled) and at end of program (black, flat line).
Figure 34 Drift of signal with time, flat-line compared to calibration point (shutter that blinds optics). Absolute value comparable to Figure 33.

\section{Error Sources}

The MES chip has many metal-metal connections with some at high temperature. Ni-Ti-Pt is present on the filament, $\mathrm{Au}-\mathrm{Ni}$ at the bond pad, and gold to aluminum or copper on the external mounting. These introduce thermocouple potential errors. To zero this noise source, the filament is operated with a square wave AC signal applied for heating at about $1 \mathrm{kHz}$. As the thermal time constant of the filament is over $100 \mathrm{~ms}$, there is no appreciable cooling between these cycles. Averaging measurements over several cycles removes the thermocouple effects.

Precision resistors are required for current measurements and balancing the bridge when used. The resistors must have a temperature coefficient less than $1 \mathrm{ppm} /{ }^{\circ} \mathrm{C}$ - but such items are available commercially. Other possible errors related to these resistors are thermocouple mismatches of the leads, voltage coefficient of resistance, drift with age and self-heating. The last is important for this application. Good packaging practice (potting) to assure isothermal operation of all components can reduce thermocouple and self-heating effects, but then the potting compound can have leakage currents that vary with temperature. 
Errors may be introduced by variations of the response of integrated circuits, especially the operational amplifiers (op-amp). Variations in offset voltage, offset drift, floating voltage errors or changes in gain can affect the signal output. Characteristics for the high precision chopper stabilized op-amp LTC1051 were used to estimate the possible magnitude of this error. The thermal drift of this circuit is given as $0.05 \mu \mathrm{V} /{ }^{\circ} \mathrm{C}$. Translating this number to an equivalent error for $\mathrm{CO}_{2}$ concentration would give $0.33 \mathrm{ppm} /{ }^{\circ} \mathrm{C}$. If the electronics are temperature stabilized this is negligible. The open loop gain of this amplifier is $10^{6}$ representing a temperature independent error of $1 \mathrm{ppm}$ full scale reading. For those measurements requiring a floating amplifier, the pixel and set point resistance, the LTC2053 circuit can be used with similar implications for final total error - under $1 \mathrm{ppm} \mathrm{CO}_{2}$ concentration error per degree change in circuit temperature. These induced errors are small compared to stated accuracy of $\pm 50 \mathrm{ppm}$.

\section{Gas Testing}

\section{Carbon Dioxide Measurements}

Sensors used for gas testing were similar in design and packaging to that shown in Figure 31. The MEMS chip was packaged in a leadless chip carrier (LCC) with sapphire window. One of the filaments was "blinded" by a gold film deposited on this window (not visible in picture). The sensor was placed in a carrier mounted on a printed circuit board opposite a spherical mirror about $2.5 \mathrm{~cm}$ from the surface of the LCC. The printed circuit board (PC) was then placed in a sealed, temperature controlled box. The atmosphere inside this box was changed by external control. Because the volume of this box was finite and well suited to rapid flow the time to change composition to a steady state was on the order of a few minutes. All electrical connections were sealed. Box temperature was typically $30^{\circ} \mathrm{C}$, just above room temperature.

Prior to use for this test, the sensors as mounted in the LCC were "calibrated" by placing them in an oven (inert atmosphere) and monitoring the resistance of the filaments as the temperature was raised from $30^{\circ} \mathrm{C}$ to over $350^{\circ} \mathrm{C}$. This pre-test provided a resistance versus temperature calibration curve. The test was performed quickly so as not to age or cause drift of the sensor. This data allowed setting the temperature of the two filaments to be equal based upon measure of the electrical resistance. Note that pre-measuring devices at room temperature before sealing allowed choice of sensors with filaments closely matched in resistance (within 1\%). Filament temperature was $350^{\circ} \mathrm{C}$ for these tests.

The source of gas in the test cell (aside from ultra-high purity nitrogen) was mixture of $20 \% \mathrm{CO}_{2}$ diluted in $\mathrm{N}_{2}$. The mixture was measured by the supplier to a NIST calibrated accuracy of $0.1 \%$. This gas was diluted, using electronic mass flow controllers (NIST traceable accuracy to $\pm 0.5 \%$ ), to successively lower concentrations of carbon dioxide of $10 \%, 5 \%, 1 \%, 0.5 \%, 0.25 \%, 0.1 \%$, $0.05 \%$ and $0.01 \%(100 \mathrm{ppm})$. Different flow controllers from $10 \mathrm{slpm}$ to $100 \mathrm{sccm}$ were used to achieve this spread in concentration. The response of the sensor to step changes in carbon dioxide concentration is shown in Figure 35. The sensor can clearly resolve a step change of $100 \mathrm{ppm}$ without ambiguity above background noise, and with time averaging, down to 50ppm. This data was repeatable for different sensors.

The vacuum was quite good in this device ( $<20 \mathrm{mTorr})$ and the resistance match between the two pixels was within $0.5 \%$. Long term stability measurements were carried out after initial gas tests to determine signal size, signal to noise ratio, and drift. During the testing fluctuations in room temperature of over 5 degrees also provided insight into the temperature stability of the device. 
Following is a summary of results and what they mean in reference to potential indoor air quality (IAQ) markets. This is raw data with 1-second update time. No smoothing, filtering, or other data processing has been implemented so that we get the best assessment of the true performance and then enhancements can be made to improve the system.

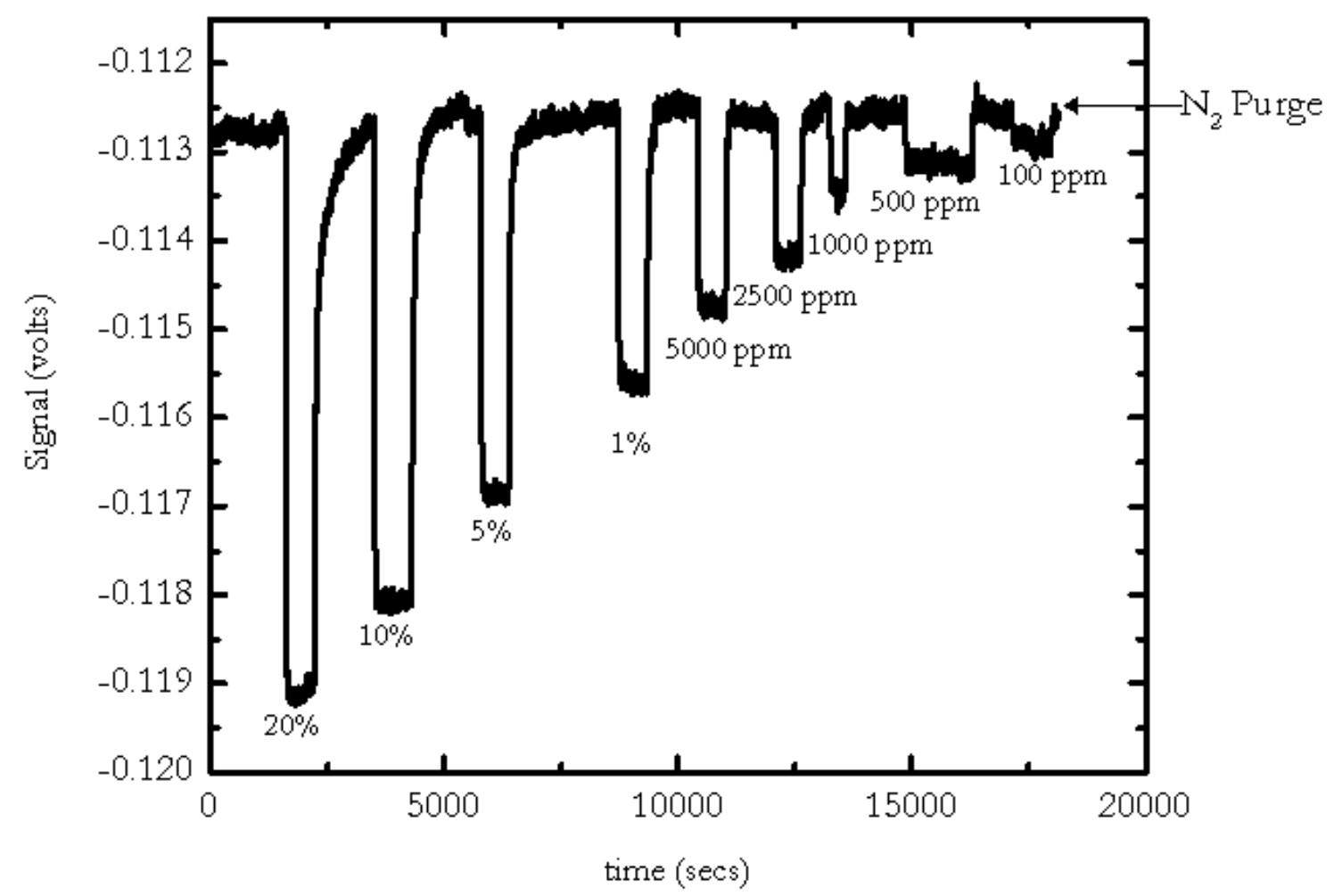

Figure 35 Ion Optics SensorChip ${ }^{\mathrm{TM}}$ precision for $5 \mathrm{~cm}$ path length gas cell $\mathrm{CO}_{2}$ in diluted in high purity nitrogen. Sensitivity to 100ppm demonstrated.

Minimum resolution=100 ppm @ 1 sec updates with a $\mathrm{S} / \mathrm{N}$ (signal to noise ratio) of 2 Range $=0.01 \%-20 \% \mathrm{CO}_{2}$ in $\mathrm{N}_{2}$ (non-linear, higher concentrations means lower resolution) Baseline Stability $=0.3 \%$ Full Scale Gas/day $=100 \mathrm{ppm} /$ day $\left(\mathrm{FSG}\right.$ was $\left.20 \% \mathrm{CO}_{2}\right)$

Temperature Stability $=0.01 \% \mathrm{FSG} /{ }^{\circ} \mathrm{C}=2 \mathrm{ppm} /{ }^{\circ} \mathrm{C}$

Compare these results to indoor Air Quality requirements:

Resolution required $=50 \mathrm{ppm}\left(\mathrm{CO}_{2}\right.$ in air $)$

Range $=50$ ppm -2000 ppm @ 50 ppm steps

Baseline Stability $=50 \mathrm{ppm} /$ day

Temperature Stability $=0-50{ }^{\circ} \mathrm{C}$

Extrapolating the data collected and using our device models, Ion Optics existing sensor can meet IAQ specs with a $10-15 \mathrm{~cm}$ path length. This would increase the signal and give 50-ppm baseline resolution and 50-ppm step changes. With a 30-second data update time signal averaging reduces the noise by a factor of 3 , further improving sensitivity. With increased signal, the identical electrical noise voltage equates to the $50 \mathrm{ppm}$ per day stability. 


\section{Interference from other vapors}

Emission from the photonic crystal (Figure 6) has a sharp peak but also contains a broad, lowlevel background that overlaps absorption lines of many other vapors. In particular, the sensor will detect water vapor and high concentrations of volatile organics as might be present if the interior room sampled were being painted or cleaned. Ion Optics examined the effect of these interferents. ${ }^{16}$ A correction must be made for relative humidity, but as this data is always measured for indoor air quality additional instrumentation is not needed. The correction is applied in software during data analysis. The signal from volatile organics detected by the $\mathrm{CO}_{2}$ monitor is sufficiently small that it can be ignored.

To test the effect of humidity on the sensor signal, water vapor was added to the diluent gas (high purity nitrogen) in a controlled fashion using a bubbler. The nitrogen was bubbled through water held at a temperature $5^{\circ} \mathrm{C}$ below room temperature (about $15^{\circ} \mathrm{C}$ ). The space above the water is saturated with water vapor in equilibrium at that temperature. This vapor is passed through tubing at a slightly higher temperature $\left(23^{\circ} \mathrm{C}\right)$ to prevent condensation in the transport lines to the experiment. The sensor runs in a closed box at $30^{\circ} \mathrm{C}$ also to prevent condensation. The relative humidity (RH) was initially $100 \%$ in the bubbler, but drops as the temperature was raised. The RH was separately measured. Data are presented in Figure 36.
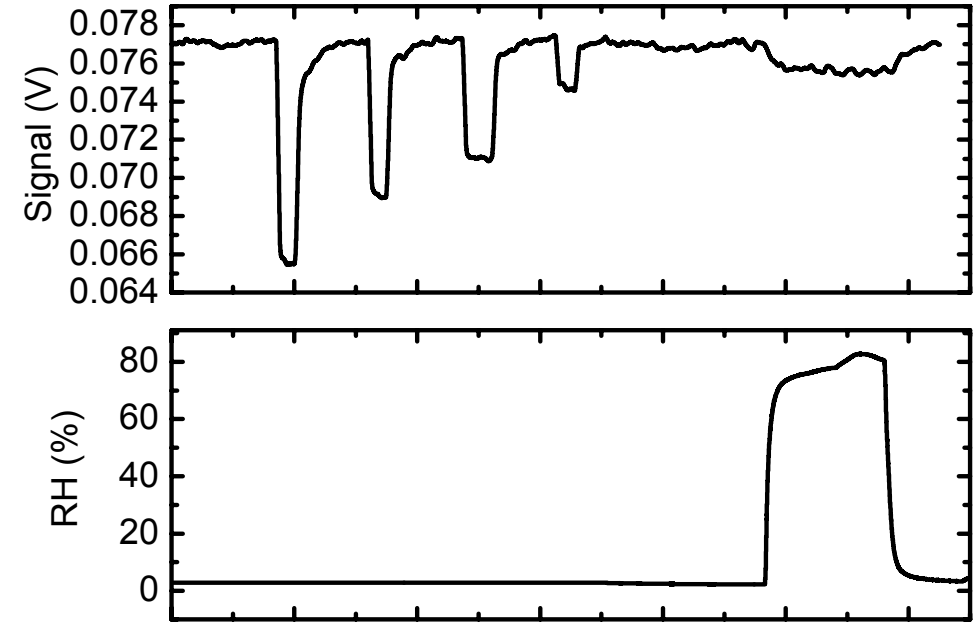

Time $(A U)$
Figure 36 The effect of water vapor on $\mathrm{CO}_{2}$ measurements with breadboard sensor. Top graph is sensor output (left to right) for $5 \%, 1 \%, 0.5 \%$ and $0.1 \% \mathrm{CO}_{2}$ in $\mathrm{N}_{2}$, plus water vapor signal. Bottom graph is time correlated RH. Data show interferent signal detectable. Signal correction available for high precision.

The signal from water vapor is as reproducible as signals from carbon dioxide measurements. Therefore, a sensor can be calibrated for $\mathrm{H}_{2} \mathrm{O}$ and a correction applied in digital analysis software. For lower levels of humidity, close to those considered comfortable, the correction is smaller and any errors in this calculation are not significant compared to cut-offs used for building control algorithms.

Two experiments were conducted to ascertain the interference from hydrocarbon vapors; one used methane (e.g. what happens in case of a natural gas leak) and one used alcohol vapor to simulate cleaning solvents. The effect of methane can be ignored. Data in Figure 37 show no significant signal for a high concentration (1\%) of methane diluted in nitrogen. Data from alcohol is shown in Figure 38. For reference, the concentration of alcohol in breath of a very 
drunk person is about 500ppm and that powerful odor would only register as an extra $100 \mathrm{ppm}$ of $\mathrm{CO}_{2}$ so this effect is also a very small correction that can be ignored for the intended application.

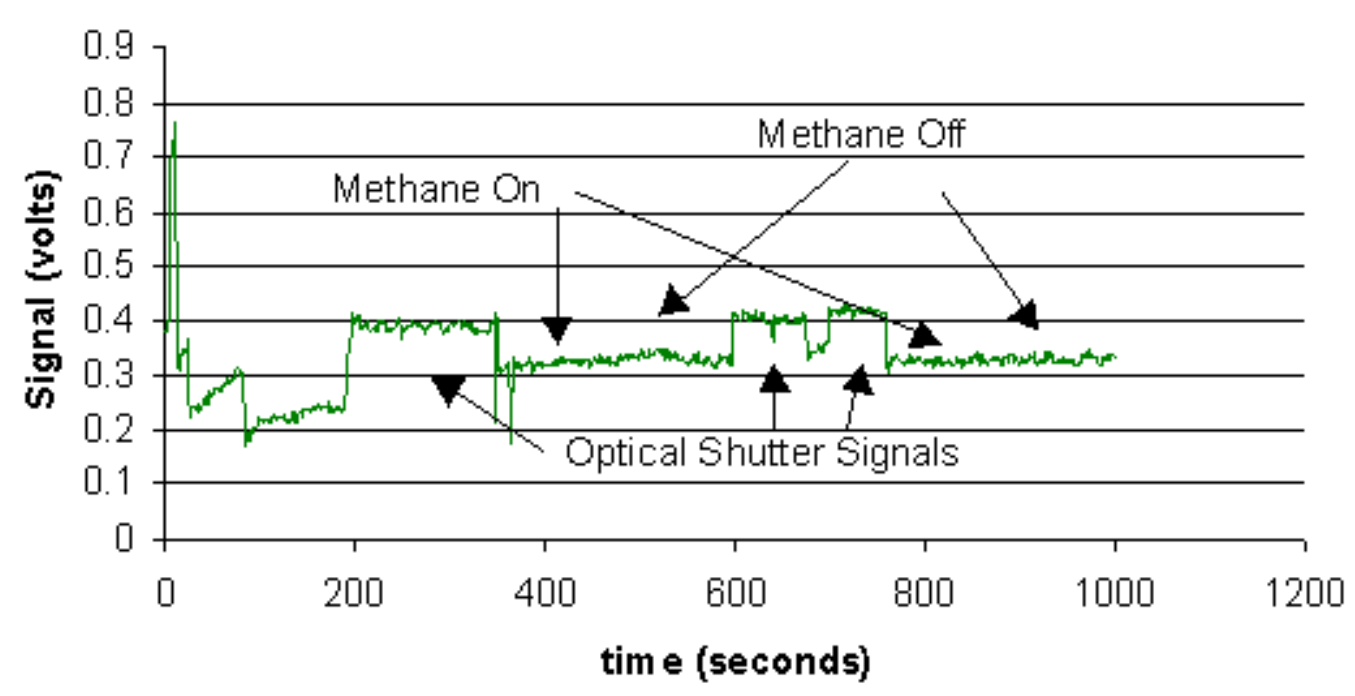

Figure 37 Signal from MEMS carbon dioxide sensor for simulant of potential interferent hydrocarbons. Methane concentration was $1 \%$ in nitrogen. Signal is lost in the noise.

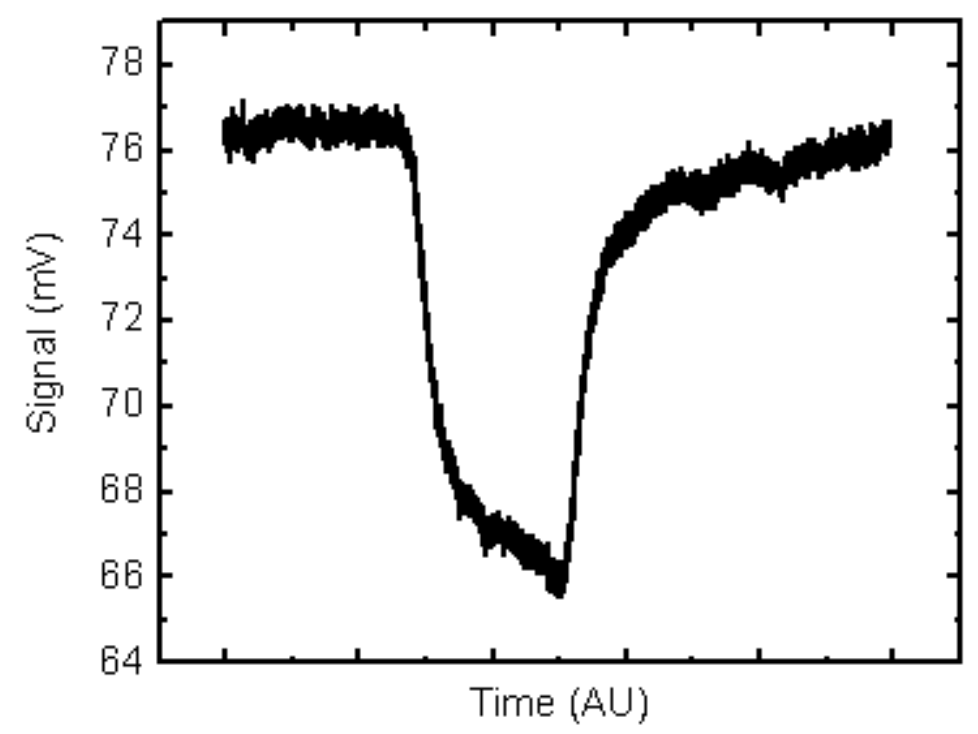

Figure 38 Signal from alcohol as a potential interferent. Sensitivity about 1/5 that for $\mathrm{CO}_{2}$ compared to that of Figure 36. 


\section{CONCLUSIONS / SUMMARY}

Ion Optics, Inc. has developed a MEMS chip that can sense carbon dioxide with sufficient precision for use as an indoor air quality measure. Other properties of this device including power requirements, calibration drift with temperature or time, interference effects of other vapors, and longevity are well within technical specifications of potential OEM customers. Initial prototype samples have been delivered to some of these customers. Widespread adoption of such sensors can be used to automate building ventilation systems in the on-demand mode with significant energy savings.

The single remaining engineering issue is a reduction of packaging costs. The prototype package shown in Figure 5 costs over $\$ 100$ each because of parts cost - the leadless chip carrier (LCC), the sapphire window with infrared antireflection coatings, getter coatings, wire bonding and chip bonding. Independent of DOE funding Ion Optics is working on a potential solution - whole wafer bonding that produces a complete package prior to dicing chips from the wafer. The first effort at this new package is shown in Figure 39. The MEMS chip has a silicon top cover that acts as a seal and IR window. It is much smaller than the LCC but was mounted in one for electrical connections in preliminary tests. In production it can be mounted on a PC board directly at a cost under $\$ 15$ each. This effort will allow us to meet the cost targets of customers.

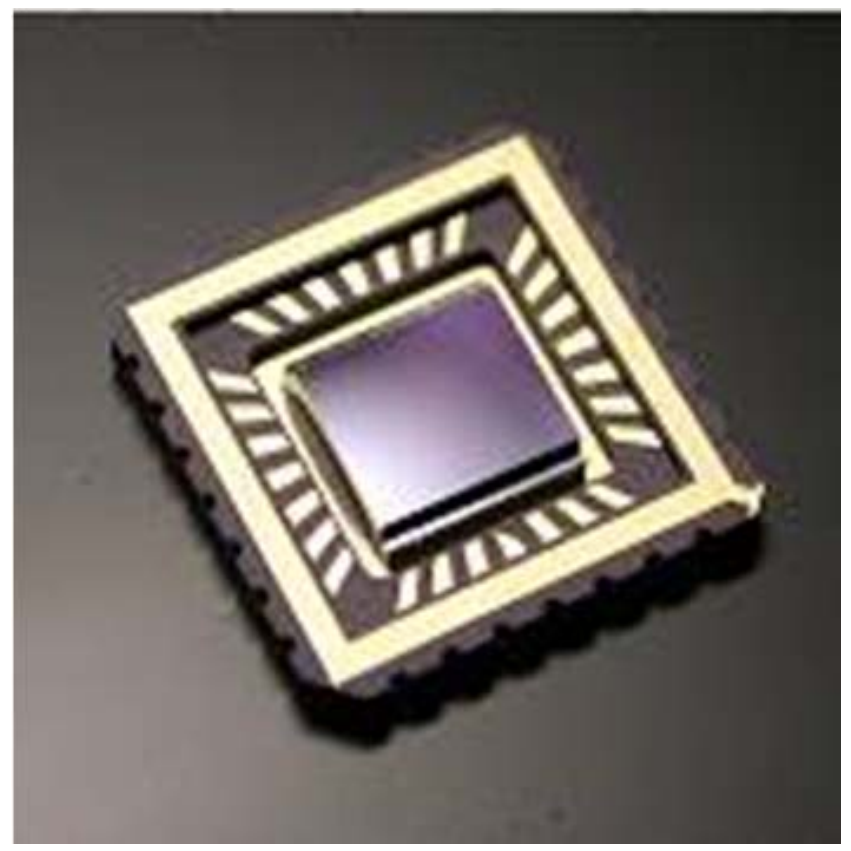

Figure 39 MEMS chip in wafer level package attached to LCC (only for electrical connections in test). 


\section{PRESENTATIONS AND PUBLICATIONS}

-Related work funded by NSF was published in the reference below. The original author was Dr. Biswas of Iowa State University who reported his earlier calculations on Ion Optics 2-D photonic crystal structures used for the gas sensor we are developing under this program.

"Tunable narrow-band infrared emitters from hexagonal lattices", I. El-Kady , R. Biswas , Y. Ye, M.F. Su, I. Puscasu, Martin Pralle, E.A. Johnson, J. Daly, A. Greenwald, Photonics and Nanostructures - Fundamentals and Applications, Vol 1(1), p69-77 (December 2003).

-A technical presentation at SPIE was given in August 2004. The paper was published in conference proceedings.

Irina Puscasu, Edward A. Johnson, Martin U. Pralle, Mark P. McNeal, James T. Daly, and Anton C. Greenwald, "Photonic crystals enable infrared gas sensors", Proceedings of SPIE -- Volume 5515, Nanoengineering: Fabrication, Properties, Optics, and Devices, Elizabeth A. Dobisz, Louay A. Eldada, Editors, October 2004, pp. 58-66.

- Two papers were presented at the Materials Research Society meeting in Boston November 29 through December 3. The work will not be published in meeting proceedings as Ion Optics intends to submit papers to refereed journals. Both oral presentations acknowledged DOE support. One of the symposia at this meeting was specifically dedicated to sensors and especially, though not exclusively, to gas sensors. Notable data relevant to this work was that no presented technology for sensing $\mathrm{CO}_{2}$ would be economically competitive with the technology being developed by Ion Optics.

- A presentation was given by Ion Optics personnel at a medical conference on detection of gases in exhaled breath. We are seeking NIH support for development of a modified sensor for this application for detection of $\mathrm{CO}_{2}$ and hydrocarbons. DOE support was acknowledged and proceedings are not published. 


\section{LIST OF ACRONYMS AND ABBREVIATIONS}

\begin{tabular}{|c|c|}
\hline 2-D & two dimensional \\
\hline $\mathrm{AC}$ & alternating current \\
\hline A-D & analog to digital \\
\hline Amp & amplifier \\
\hline ASHRAE & American Society for Heating, Refrigeration and Air-Conditioning Engineers \\
\hline AU & arbitrary units (uncalibrated scale) \\
\hline DCV & demand controlled ventilation \\
\hline DOE & Department of Energy \\
\hline DRIE & deep reactive ion etching \\
\hline DSP & digital signal processor \\
\hline FEM & finite element modeling \\
\hline FWHM & full width at half-maximum \\
\hline HVAC & heating, ventilation and air conditioning \\
\hline IAQ & indoor air quality \\
\hline $\mathrm{IC}$ & integrated circuit \\
\hline IR & infrared \\
\hline $\mathrm{kHz}$ & kilo-hertz \\
\hline LCC & leadless chip carrier (package) \\
\hline MEMS & micro-electrical mechanical system(s) \\
\hline $\mathrm{mm}$ & millimeters \\
\hline $\mathrm{ms}$ & millisecond \\
\hline mtorr & milli-torr \\
\hline $\mathrm{mW}$ & milli-watts \\
\hline $\mathrm{nm}$ & nano-meters \\
\hline NIST & National Institute of Standards and Technology \\
\hline NIH & National Institute of Health \\
\hline NSF & National Science Foundation \\
\hline $\mathrm{OP}$ & operational amplifier \\
\hline$\Omega-\mathrm{cm}$ & ohm-centimeters \\
\hline PBG & photonic band gap \\
\hline PC & printed circuit \\
\hline ppm & parts per million \\
\hline RTD & resistance temperature detector \\
\hline SEM & scanning electron microscope \\
\hline SOI & silicon-on-insulator \\
\hline TEM & transmission electron microscope \\
\hline $\mathrm{um}$ or $\mu \mathrm{m}$ & micron \\
\hline$\mu \mathrm{V}$ & micro-volt \\
\hline VAV & variable air volume \\
\hline
\end{tabular}




\section{REFERENCES}

1 Data courtesy Jim Sand of ORNL, DOE government laboratories.

2 DeAlmeida and Fisk, "Sensor-Based Demand Controlled Ventilation", technical report LBNL40599 (1997).

3 W. J. Fisk and A. T. DeAlmeida, Energy and Buildings, 29,p35-45, (1998).

4 U.S. Patent Application number 09/762,077

5 U.S. Patents 6,373,056 and 6,528,792

6 I. El-Kady, R. Biswas, Y. Ye, M.F. Su, I. Puscasu, Martin Pralle, E.A. Johnson, J. Daly, A. Greenwald, "Tunable narrow-band infrared emitters from hexagonal lattices", Photonics and Nanostructures - Fundamentals and Applications, Vol 1(1), p69-77 (December 2003). Also

Irina Puscasu, M. Pralle, M. McNeal, J. Daly, A. Greenwald, E. Johnson, R. Biswas and C. G. Ding, "Extraordinary emission from two-dimensional plasmonic-photonic crystals", J. Appl. Phys., 98, p13531 (2005).

7 J. Dintinger, A. Degiron and T.W. Ebbesen, "Enhanced Light transmission through Subwavelength Holes", MRS Bulletin, v30, p381 (May 2005).

8 Data from Ion Optics internally funded effort prior to start of DOE program.

9 Mark McNeal, James T. Daly, Anton C. Greenwald, Edward A. Johnson, Nicholas Moelders, Martin Pralle, Thomas George and Daniel S. Choi, "Thermal Losses And Temperature Measurement In SOI MEMS Heater,” Materials Research Society Symp. Proc. v687, (2002).

10 Semancik, Cavicchi, Gaitan, and Suehle, U.S. Patent 5,345,213 (1994).

11 National Science Foundation Phase II SBIR grant entitled: "Photonic Crystal Coherent Thermal Emission for Sensors" number DMI-0450397, Principal Investigator Dr. Irina Puscasu.

12 www.ansys.com

13 www.comsol.com

14 www.algor.com

15 DOE grant number DOE-FG02-ER83203, "Compact, Rugged, Low-Power CO2 Sensors For Balloon-Borne Measurement Of Atmospheric CO2 Concentration".

16 Funding for measurement of interferent effects provided by Ion Optics. Research also reported for NIH grant 1-R43-ES11691-01 "Disposable CO2 Sensor for Emergency Resuscitation". 